\title{
Outage Performance of the Primary Service in Spectrum Sharing Networks
}

\author{
Mohammad G. Khoshkholgh, Keivan Navaie, Senior Member, IEEE, and \\ Halim Yanikomeroglu, Senior Member, IEEE
}

\begin{abstract}
In this paper, we utilize stochastic geometry to analyze the primary service (PS) outage performance for spectrum sharing in Rayleigh fading environment. Using this approach, the impacts of the secondary service (SS) parameters and wireless environment on the PS outage probability are analyzed. We further obtain a closed form for the PS outage probability. The maximum SS transmitter node density for a given outage probability constraint of the PS is then obtained. We also investigate the impact of secondary spectrum sensing on the PS outage probability. A novel approach is further proposed that provides tight approximation for the PS outage probability. The results of the proposed approach are then validated through analysis and simulations. We then consider power control in the secondary network and show that the truncated channel inversion power control significantly decreases the PS outage probability. Cases with centralized and decentralized cooperative spectrum sensing are also studied, and their corresponding PS outage probabilities are analyzed. Mean spatial throughput of the SS is also analyzed. We further investigate the impact of the PS outage constraint on the spatial throughput of the SS. Extensive simulations confirm our analytical derivations.
\end{abstract}

Index Terms-Outage probability, spectrum sensing, spectrum sharing, stochastic geometry

\section{INTRODUCTION}

$\mathrm{T}$ O improve the utilization of the allocated frequency bands, spectrum sharing is proposed by the Federal Communications Commission [1]. In this method, under certain conditions, a secondary service (SS) is able to access to a frequency band formally allocated to the primary service (PS) [2], [3]. Various schemes are proposed in the literature for spectrum sharing (see, e.g., [4]) where they are usually referred to as the dynamic spectrum access (DSA).

In the DSA, the SS dynamically detects and utilizes the spectrum holes or white spaces [3], [6]. White spaces are those parts of the spectrum allocated to the primary user that are underutilized in some particular times and specific locations. Here, we focus on the overlay spectrum sharing that is also referred to as the opportunistic spectrum access (OSA) [4], [5].

In the OSA, the SS utilizes spectrum sensing schemes to detect the white spaces. If the spectrum condition is detected as "idle" (i.e., the PS is inactive), the SS is allowed to transmit its own information; otherwise (i.e., the PS is active), the SS is not allowed to transmit. Therefore, given perfect spectrum sensing, adopting OSA increases the spectrum efficiency while imposes no negative impact on the PS.

- M.G. Khoshkholgh is with the Simula School of Research and Innovation AS Network Systems, PO Box 134, 1325 Lysaker, Norway.

E-mail: khoshkholgh@simula.no.

- K. Navaie is with the School of Electronic and Electrical Engineering, University of Leeds, Leeds LS2 9JT, United Kingdom.

E-mail:k.navaie@leeds.ac.uk.

- H. Yanikomeroglu is with the Department of Systems and Computer Engineering, Carleton University, 1125 Colonel By Drive, Ottawa, Ontario K1S 5B6, Canada. E-mail: Halim.Yanikomeroglu@sce.carleton.ca.

Manuscript received 25 Dec. 2010; revised 6 Feb. 2012; accepted 1 July 2012; published online 10 July 2012.

For information on obtaining reprints of this article, please send e-mail to: tmc@computer.org, and reference IEEECS Log Number TMC-2010-12-0587. Digital Object Identifier no. 10.1109/TMC.2012.156.
In practice, however, spectrum sensing schemes are usually imperfect [3], [4]. The inaccuracy in the spectrum sensing results in degrading quality of service (QoS) of the PS users. Furthermore, by increasing the number of the SS users, the spectrum sensing inaccuracy may seriously affect the performance of the primary network. Therefore, in the spectrum sharing, it is essential to manage the SS parameters such that the PS QoS constraints including the outage probability and achievable capacity are always kept satisfied.

One of the main parameters to be adjusted is the maximum number of the SS users in a given coverage area of the primary network. To obtain the maximum number of the SS users, in this paper, we employ stochastic geometry (see, e.g., [7]) that is shown to be a very powerful mathematical tool for performance evaluation of wireless networks (see, e.g., [8], [9], [10], [11], and [12]). A comprehensive tutorial on the stochastic geometry and its application in wireless communication networks can be found in [13] and [14].

In [15], based on homogenous Poisson point process (PPP), upper and lower bounds on the transmitter node density are obtained to satisfy the outage probability constraint in code division multiple access-based ad hoc networks. The impact of fading, power control, and interference cancelation on the outage probability in ad hoc networks are also studied in [16] and [17]. Stochastic geometry has been also successfully adopted for connectivity modeling in ad hoc networks [18]. In [19], [20], the authors utilize advanced stochastic geometry to design efficient hierarchical sensor networks with the objective of minimizing the network energy consumption.

In the related literature, stochastic geometry is also employed to analyze spectrum sharing performance. Channel capacity of the SS is the focus of Zhang et al. 
[21]. Furthermore, in [22], a distance-dependence path-loss attenuation model is considered, and log-normal distribution is proposed as an approximated probability distribution function of the aggregate interference at the PS receiver. Considering fading effects of the wireless channel as well as spectrum sensing parameters, Ghasemi and Sousa [23] propose an approximation for the distribution of the interference aggregation at the PS receiver. They also analyze the impact of cooperative spectrum sensing among the SS users on the PS outage probability. However, they assume that the SS transmitters are able to identify the spectrum status using signaling channels among the PS receiver and the SS transmitters. Further their work is restricted to calculate the cumulates of the aggregate interference at the PS receiver.

The PS outage probability is also investigated in [22], [23]; however, they do not provide closed-form approximations. In [24], [25], the authors extended the framework presented in [26] into underlay spectrum sharing. Moreover, in [27], assuming deterministic exclusion regions around the PS nodes, upper bounds were obtained for the outage in the PS receiver for underlay spectrum sharing. Since the deterministic exclusion counter considered in [24], [25], [27], is a direct result of distant-dependence path loss, by considering fading/shadowing, it is not valid anymore. Instead of that, amoebous-like counter might be more realistic.

In this paper, we extend the perspective presented in [22] and [23] to obtain a closed form for the PS outage probability and investigate the impact of the power control. Moreover, we present a useful approach to obtain an approximation of the PS outage probability. We further show that the proposed approximation follows the actual value closely. We then obtain the maximum density of the SS transmitters corresponding to a given PS outage probability.

Stochastic geometry has been adopted in the related literature to shed light on the performance of the spectrum sharing systems and design new access strategies. In [28], utilizing the stochastic geometry, a spectrum sharing scenario is developed in which the secondary system is a mobile ad hoc network, and the primary system is a cellular network with orthogonal frequency-division multiplexing technology. In [28], upper and lower bounds on the PS outage probability are also obtained. Moreover, in [29], utilizing stochastic geometry for modeling of the primary network, the impact of the PS transmitter density as well as the SS power control on the spectrum sensing performance is analyzed. The system model adopted in this paper is different from those are considered in [28] and [29].

In this paper, utilizing stochastic geometry approach in modeling the spectrum sharing systems, we obtain a closedform formula for the PS outage probability. In addition to the distance-dependent path-loss attenuation that is considered in the previous studies, we also consider fading in our modeling. In addition, in our analysis, we also consider inaccurate spectrum sensing. Inaccurate spectrum sensing is modeled through a receiver operating characteristic (ROC) curve that relates miss detection and false-alarm probabilities. The maximum density of the SS transmitters is then obtained based on the PS outage probability as well as the primary and SS parameters.
We also obtain a tight lower bound for the PS outage probability. The validity of the obtained lower bound is also investigated through analysis as well as numerical studies. The obtained lower bound is similar to the one presented in [16]; however, we show that the outage bound can be obtained merely by considering the particular secondary transmitter that creates the maximum imposed interference at the PS receiver. We further observe that in the obtained lower bound, by substituting the SS transmitter density, $\lambda_{s}$, with $\left(1+a_{m}(\alpha)\right)^{\frac{2}{\alpha}} \lambda_{s}$, the lower bound is transformed to a good approximation of the actual PS outage probability, where $\alpha$ is the path-loss exponent and $a_{m}(\alpha) \in[0, \mathcal{C}(\alpha))$ is an adaptive parameter. Here, $\left.\mathcal{C}(\alpha)\right)$ is a deterministic function of $\alpha$, where utilizing asymptotic analysis has been conducted in [16], which is obtained as $\mathcal{C}(\alpha))=\left(\alpha \log _{2}\left(\frac{\alpha}{\alpha-1}\right)-1\right)^{+}$. We also show that this approach is also applicable to log-normal shadowing as well as Rayleigh fading environments. Acceptable level of accuracy and its simplicity are the main advantages of the proposed approach. In comparison to [16], [17], and [26], we obtain a tighter and at the same time simpler approximation for the outage probability.

Further, we include the PS power control in our modeling and analyze its impact on the PS outage probability. We show that the SS power control based on truncated channel inversion significantly decreases the PS outage probability. We further analyze the impact of cooperative spectrum sensing. We consider both centralized and decentralized cooperative spectrum sensing. We then show that in the centralized cooperative spectrum sensing, the density of SS transmitters that experience miss detection approaches to zero. It is also shown that in the decentralized cooperative spectrum sensing, increasing the density of the SS transmitters results in more accurate spectrum sensing and, thus, decreases the corresponding negative impact on the PS.

Here, we also investigate issues regarding the SS performance particularly the mean spatial throughput. Appropriate optimization problems for evaluating maximum spatial throughput in cases with/without power control and cooperative sensing are presented. We then examine the impact of the secondary transmitter density and the PS outage constraint on the spatial throughput of the SS. Moreover, we show that truncated power control mechanism can enhance the spatial throughput of the SS.

The analysis provided in this paper enables us to recognize the essential SS parameters that by adjusting them a certain level of QoS can be guaranteed in the primary network. We also confirm our analytical results through comparing them with simulation results. Simulation results are followed closely by the analytical results, particularly for the case that the SS node density is sufficiently high.

Some simplistic assumptions are made in this paper. First of all, our analysis is generally focused on the homogenous PPP. Cases including nonhomogeneity or clustered point process are not generally in the scope of this paper. Second, we have assumed that the separation distance between the secondary peers is fixed and the same for all nodes. ${ }^{1}$ Moreover, we only examine the outage

1. This assumption is only considered in Section 5; however, results in [16] and [15] indicate that the general trends in the case of random separating distance may not dramatically vary. 
probability on a typical primary receiver. In practice, a network of primary receivers exists. ${ }^{2}$

The rest of this paper is organized as follows: Section 2 presents the system model. In Section 3, the PS outage probability is obtained, and the impact of spectrum sensing on the PS outage probability is addressed. A new approach to approximate the PS outage probability is developed in Section 4 . Section 5 covers the analysis of the impact of the SS power control strategy on the PS outage probability. Centralized and decentralized cooperative spectrum sensing are also analyzed in Section 6 . The SS performance is investigated in Section 7. In Section 8, we compare the analytical results with the simulations. Conclusions are provided in Section 9.

\section{System Model}

In spectrum sharing, two services try to access a $B \mathrm{~Hz}$ spectrum band: the $P S$, and the SS. The spectrum has been licensed to the PS. The SS does not have the spectrum license, but may acquire access to the spectrum by adopting OSA. The system model we consider in this paper was successfully examined in the previous works see, for example, [22], [23].

\subsection{Channel Model}

The wireless channel is an additive white Gaussian noise (AWGN) with flat fading. AWGN power spectral density is $N_{0}$. The propagation power loss in this paper is modeled based on a loss function, $l(x, y)$, which returns the path loss between locations $x=\left(x_{1}, x_{2}\right)$ and $y=\left(y_{1}, y_{2}\right), x, y \in \mathbb{R}^{2}$. In general, $l(x, y)$ is a function of

$$
\|x-y\| \triangleq \sqrt{\left(x_{1}-y_{1}\right)^{2}+\left(x_{2}-y_{2}\right)^{2}} .
$$

In this paper, we consider distance-dependent path loss, where $l(x, y)=\kappa\|x-y\|^{-\alpha}$ [9], [15]. Parameter $\alpha>2$ is the path-loss exponent, and $\kappa$ is a constant that is a function of wireless channel propagation profile as well as the antenna characteristics at the transmitter and the receiver. For brevity, in the rest of this paper, we assume $\kappa=1$. For $x=y, l(x, y) \rightarrow \infty$. Since our main focus is to evaluate the probabilistic behavior of the network, this singularity does not have major effect on the obtained results. This assumption has been also made in the related literature, see, for example, [16] and supported by the simulation results presented in Section 8.

Fading is modeled as a random variable (r.v.) $g$ with probability density function (pdf), $f_{g}(g)$, where $g$ is power gain. Here, we assume that $\sqrt{g}$ is a Rayleigh r.v.; therefore, $g$ is an exponential r.v. with mean value $1 / \mu$, and $f_{g}(g)=$ $\mu e^{-\mu g} U(g)(\mathrm{U}($.$) is the step function) [30]. Based on the$ aforementioned wireless channel model, the ratio of transmitted power to the received power in a location with distance $u$ from the transmitter is $u^{-\alpha} g$. We further assume that $g$ is independent across different users and at the different locations.

\subsection{Spectrum Sensing and a Network Model}

Consider a single PS transceiver where the receiver is located at the center. We assume that the distance between

2. Noting the Palm distribution in stochastic geometry [7], this assumption can be justifiable. the primary transmitter and receiver is $R>1$. The PS transmits with a fixed power transmission power, $S_{p}$. The fading in the channel between the primary transmitter and receiver is $g_{p}$. Therefore, the received power at the primary receiver is equal to $S_{p} R^{-\alpha} g_{p}$.

The primary transmitter is surrounded by an SS ad hoc network. The secondary users acquire access to the spectrum by adopting OSA. Each secondary node senses the spectrum and if it is idle, the corresponding SS transmitter starts transmission. Transmission is stopped immediately if PS activity is detected.

The spectrum sharing efficiency is significantly affected by the performance of spectrum sensing. The performance of the spectrum sensing is determined by two important parameters namely probability of false alarm, $\epsilon$, and probability of miss detection, $\delta$ [4]. If the spectrum is idle and the spectrum sensing wrongly recognize the spectrum status as busy, a false alarm is occurred. Furthermore, miss detection is occurred in cases where the spectrum is busy, and it is mistakenly recognized as idle. A spectrum sensing with high probability of false alarm results in a lower corresponding achieved SS capacity. This is due to the fact that the false alarm limits the secondary access to the actual idle periods of the spectrum. Miss detection results in imposing interference on the PS by the secondary transmission that may result in an increase in the PS outage probability. The ideal sensing procedure is the one with zero false alarm and miss detection probabilities.

In practice, however, the false alarm and miss detection probabilities are related to each other through an ROC curve, which is a fundamental attribute of each spectrum sensing system [4]. The ROC curve usually relates the detection probability, i.e., $1-\delta$, to $\epsilon$. Here, for brevity of expositions, we first assume that the ROC curve is identical for all of the SS users. Cases including distant-dependent ROC are demonstrated in Section 3.3.

Let the SS users be distributed on a plane based on a homogenous PPP with density $\lambda_{s}$. Therefore, for a given region, $\mathcal{R}$, with area $A \mathrm{~m}^{2}$, the probability of having $k$ active $^{3}$ SS transmitters is obtained as

$$
\mathbf{P}\{k \quad \text { in } \quad \mathcal{R}\}=e^{-\lambda_{s} A} \frac{\left(\lambda_{s} A\right)^{k}}{k !} \quad k=1,2, \ldots
$$

Based on this spatial distribution model, the number of the SS transmitters for two disjoint regions $\mathcal{R}_{1}$ and $\mathcal{R}_{2}$ are independent r.v.s [7].

In our modeling, similar to the previous works, for example, [15], [22], the distance between an SS transmitterreceiver pair is assumed to be a fixed value. The SS transmission power is also assumed to be fixed. Later, in this paper, we extend our analysis to the case where the SS adopts a power control strategy.

\section{PS Outage Probability}

Here, we analyze the impacts of the activity of the SS users on the PS performance. In particular, our objective is to

3. The SS users that are sensing the spectrum and have traffic in the queue ready to be transmitted are referred to as active secondary users. 
identify how the density of the SS transmitters affect the PS outage performance.

Miss detection results in SS transmission during the activity period of the PS that imposes interference at the PS receiver. The aggregate interference may result in the PS outage. Therefore, the aggregate interference at the PS receiver is an important measure to model the impact of miss detection on the performance of the PS.

\subsection{Aggregate Interference}

Assume that the primary receiver is located at the origin and surrounded by secondary users. Let $\tilde{\Pi}_{s}=\left\{X_{i}, i \geq 1\right\}$ be a PPP with density $\lambda_{s}$ that indicates the location of the SS transmitters. Among them, some SS transmitters experience miss detection.

Let $\breve{\Pi}_{s} \triangleq\left\{X_{i} \in \tilde{\Pi}_{s} \mid D_{i}=1, i \geq 1\right\}$ be a set including the location of the SS transmitters that experience miss detection. In this definition, $D_{i}=1\left(D_{i}=0\right)$ if the SS transmitter located at $X_{i}$ detects the spectrum status as idle (busy), while it is active. $\breve{\Pi}_{s}$ is a homogenous PPP with density $\lambda$, where $\lambda=\delta \lambda_{s}$. Corresponding to $\breve{\Pi}_{s}$, we define

$$
\Pi_{s} \triangleq\left\{\left(X_{i}, g_{s p, i}\right) \mid X_{i} \in \breve{\Pi}_{s}, i \geq 1\right\},
$$

which corresponds $X_{i} \in \breve{\Pi}_{s}$ to $g_{s p, i}$, where $g_{s p, i}$ is the fading of the wireless channel between the secondary transmitter located at $X_{i}$, and the primary receiver located at the origin. Set $\Pi_{s}$ is a homogenous PPP with density $\lambda f_{g}\left(g_{s p}\right)$ [23].

Transmissions made by the SS transmitters at $X_{i} \in \breve{\Pi}_{s}$, $i=1,2, \ldots$ impose an aggregate interference, $I_{\phi}$, at the PS receiver, where

$$
I_{\phi}=\sum_{\left(X_{i}, g_{s, i, i}\right) \in \Pi_{s}} S_{s} u_{i}^{-\alpha} g_{s p, i} .
$$

In (2), $u_{i}=\left\|X_{i}\right\|$ is the distance between the SS transmitter located at $X_{i}$ and the origin.

\subsection{The Outage Probability}

The signal-to-interference-plus-noise ratio (SINR) at the PS receiver is

$$
\mathrm{SINR}_{p}=\frac{S_{p} R^{-\alpha} g_{p}}{W+I_{\phi}}
$$

where $I_{\phi}$ is the aggregate interference defined in (2), and $W$ is a r.v. representing the AWGN noise with variance $\sigma_{W}^{2}=N_{0} B$. The outage probability of the PS user is defined as [22]

$$
P_{\text {out }}^{p}=\mathbf{P}\left\{\mathrm{SINR}_{p}<\gamma_{t h}\right\},
$$

where $\gamma_{t h}$ is the SINR threshold. The outage may be caused by high interference $I_{\phi}$ and/or deep fading (i.e., very low $g_{p}$ ).

It is worth mentioning that in the spectrum sharing literature, an interference threshold constraint, $Q$, is usually considered which should be satisfied at the PS receiver. Therefore, the aggregate interference imposed by the SS transmissions must be kept below $Q$. Consequently, as far as the secondary network satisfies the interference threshold, i.e., $I_{\phi} \leq Q$, the spectrum is accessible [23] and [31]. Using such terminology $P_{\text {int }}^{p} \triangleq \mathbf{P}\left\{I_{\phi}>Q\right\}$ namely interference probability can be considered as a performance metric that indicates the impact of the secondary activity on the primary performance [25]. In this paper, however, we mainly consider the outage probability as a metric to study the SS activity on the PS performance.

The following proposition results in a closed-form expression of the PS outage probability. The proposition is a direct result in [9, Lemma 3.1] where for completeness it is briefly stated.

\section{Proposition 1. PS outage probability is}

$$
P_{\text {out }}^{p}=1-\Phi_{W}\left(\mu \frac{\gamma_{t h} R^{\alpha}}{S_{p}}\right) e^{-\lambda R^{2}\left(\frac{S_{s_{t h}}}{S_{p}}\right)^{\frac{2}{\alpha}} L},
$$

where $\Phi_{W}(s)$ is the Laplace transform of the AWGN pdf

$$
L=\frac{2 \pi}{\alpha} \Gamma\left(\frac{2}{\alpha}\right) \Gamma\left(1-\frac{2}{\alpha}\right),
$$

and $\Gamma(x)=\int_{0}^{\infty} t^{x-1} e^{-t} d t$.

Proof. See Appendix II, which can be found on the Computer Society Digital Library at http://doi. ieeecomputersociety.org/10.1109/TMC.2012.156.

In practice, the outage probability in the PS requires to be always less than or equal to $\xi$, which is a system parameter, i.e., $P_{o u t}^{p} \leq \xi$. As it is seen in Proposition 1, different factors affect the outage probability. Here, our main focus is to study the impact of $\lambda_{s}$.

For a given system setting, when $W \ll I_{\phi}$, the following proposition provides the maximum SS node density, $\lambda_{s}^{*}$, where the outage probability constraint is held.

Proposition 2. If $W \ll I_{\phi}$, the maximum SS transmitter density, $\lambda_{s}^{*}$, which satisfies $P_{\text {out }}^{p} \leq \xi$ is

$$
\lambda_{s}^{*}=\left(\frac{S_{s} \gamma_{t h}}{S_{p}}\right)^{\frac{-2}{\alpha}} \frac{R^{-2}}{L \delta} \ln \frac{1}{1-\xi},
$$

where $L$ is defined in (6).

Proof. See Appendix III, available in the online supplemental material.

Proposition 2 provides a closed form for the maximum allowable SS transmitter density. It is also observed that for a given PS outage constraint, $\xi$, the appropriate density of the SS transmitters, $\lambda_{s}$, is related to the wireless channel condition, represented by $L$, and the PS system parameters including $S_{p}, R$, and $\gamma_{t h}$.

To satisfy a given outage constraint, it is also seen in (39) that the SS may also adjust its power transmission level, $S_{s}$, and/or $\delta$. As it is seen, by decreasing miss detection probability $\delta, \lambda_{s}^{*}$ is also increased. Thus, the SS can adopt a more accurate sensing procedure with a lower miss detection probability and/or reduce its transmission power.

An approach to decrease the miss detection probability is to increase the accuracy of the spectrum sensing by adopting cooperative spectrum sensing. In Section 6, we show that utilizing cooperative spectrum sensing is a promising technique to decrease the PS outage probability.

Another important parameter that could be adjusted to decrease the outage probability, or equivalently to increase 
$\lambda_{s}^{*}$ for a fixed outage probability, is $S_{s}$. To adjust $S_{s}$, power control can be adopted in the secondary system. In Section 5 , we analyze the impact of the power control on the PS outage probability.

Remark 1. The above discussion is also consistent with more complicated situations corresponding to the other fading distributions, for example, Nakagami- $m$ model and/or maximum ratio combining receiver in multiinput multi-output channels, where the outage probability in (33) is written as

$$
\mathbf{P}\left\{\mathrm{SINR}_{p}<\gamma_{t h}\right\}=
$$

$$
1-\int_{0}^{\infty}\left(\sum_{n \in \mathcal{N}, k \in \mathcal{K}} b_{n k} e^{-n \mu \frac{\gamma t R^{\alpha}}{S_{p}} \theta}\left(\mu \frac{\gamma_{t h} R^{\alpha}}{S_{p}} \theta\right)^{k}\right) f_{W+I_{\phi}}(\theta) \mathrm{d} \theta
$$

where $\mathcal{N}, \mathcal{K} \in \mathbb{N}$ are finite sets (see [33] for further details). However, for the cases where the wireless channel is surrounded by obstacles, i.e., log-normal shadow fading is dominant, as well as scenarios in which a power control mechanism is also adopted, a closed-form of the PS outage probability might not be obtained. Therefore, it is required to develop an analytical framework to obtain outage probability approximation with acceptable level of accuracy (see Section 4 for more details).

Remark 2. In the above discussions, we simply assume identical spectrum sensing for the SS transmitters, i.e., all have the same ROC with the same miss detection probabilities. However, as it was also mentioned in Section 2, miss detection probability may not be the same for different secondary transmitters. For a secondary transmitter located at $X_{i}$, the performance of the spectrum sensing, among other things, is a function of the distance between the PS transmitter and the SS transmitter, where the spectrum sensing is implemented. It is also related to the fading effect of the corresponding wireless channel.

In the next section, we examine the impact of spectrum sensing on the PS outage probability. Our results indicate that we can efficiently translate the practical spectrum sensing procedure to the simple model that is considered in Section 2.

\subsection{Impact of Spectrum Sensing}

In the following, we study the impact of the distance and fading issues on the outage performance of the PS. We explore energy detector and analyze the performance in Rayleigh fading. Corresponding to a system in which each secondary transmitter has its own ROC and miss detection probabilities different from others, we then propose an equivalent secondary network with identical ROC and miss detection probabilities, which has the same PS outage probability. In [10] and [23], some approximations are obtained to evaluate $\mathbf{P}\left\{I_{\phi}>z\right\}$ based on the cumulants of the aggregate interference $I_{\phi}$. They showed that shifted log-normal approximation can fairly approximate the exact pdf of $\mathbf{P}\left\{I_{\phi}>z\right\}$. However, this approach coincides with some difficulties as this may not give us a valuable

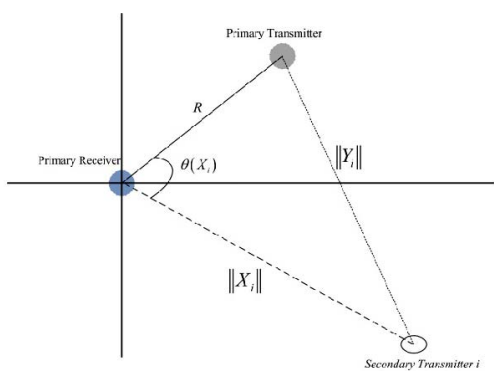

Fig. 1. A schematic diagram including the PS transceiver and an SS transmitter.

insight to figure out the impact of different parameters on PS outage probability.

Instead of this, in the following, we suggest a novel approach to exchange real spectrum sensing into the framework presented in Section 3.2 without having severe changes on the PS outage probability.

Here, to model the dependency of the spectrum sensing to the distance between the primary and secondary transmitters, we assume that the performance of the spectrum sensing is a function of the received power from the PS transmitter. As it is depicted in Fig. 1 for the $i$ th SS transmitter located at $X_{i}$, assuming $\left\|Y_{i}\right\|$ as the distance between the PS transmitter and the SS transmitter $i$, the received power of the PS transmitter is equal to $\left\|Y_{i}\right\|^{-\alpha} g_{p s, i} S_{p}$, where $\left\|Y_{i}\right\|$ is obtained as $\left\|Y_{i}\right\|^{2}=\left\|X_{i}\right\|^{2}+$ $R^{2}-2 R\left\|X_{i}\right\| \cos \left(\theta\left(X_{i}\right)\right)$ and $g_{p s, i}$ is the channel fading between the primary transmitter and SS transmitter $i$. The interference-to-noise ratio at the $i$ th SS transmitter because of the PS transmission, $\gamma_{p s, i}$, is

$$
\gamma_{p s, i}=\frac{S_{p}\left(\left\|X_{i}\right\|^{2}+R^{2}-2 R\left\|X_{i}\right\| \cos \left(\theta\left(X_{i}\right)\right)\right)^{\frac{-\alpha}{2}} g_{p s, i}}{N_{0} B}
$$

At the beginning of each time slot, each secondary transmitter $i$ measures $\gamma_{p s, i}$ and compare it with a given threshold $\gamma_{0}$. The output of the spectrum sensing is $D\left(\gamma_{p s, i}\right)$, in which $D\left(\gamma_{p s, i}\right)=1$ indicates an idle spectrum, and $D\left(\gamma_{p s, i}\right)=0$, otherwise:

$$
D\left(\gamma_{p s, i}\right)= \begin{cases}1 & \text { if } \gamma_{p s, i}<\gamma_{0} \\ 0 & \text { otherwise }\end{cases}
$$

where $\gamma_{0}$ is a SS system parameter and is related to the sensitivity of the spectrum sensing. It is well known that $\gamma_{0}$, among other things, is a function of false alarm probability. In [23], authors pointed out that for small enough false alarm probability, (9) results almost the same detection probability of the energy detector, i.e., $\delta_{i}=D\left(\gamma_{p s, i}\right)$. Remark 3 at the end of this section provides more details.

Based on (8) and (9), $D\left(\gamma_{p s, i}\right)$ is rewritten as

$$
D\left(\gamma_{p s, i}\right)=\mathbf{1}\left(\omega\left(\left\|X_{i}\right\|, \theta\left(X_{i}\right)\right) g_{p s, i}^{-\frac{2}{\alpha}}>R_{s}^{2}\right),
$$

where $R_{s}^{\alpha}=\frac{S_{p}}{\gamma_{0} N_{0} B}$ and $\omega\left(\left\|X_{i}\right\|, \theta\left(X_{i}\right)\right)=\left\|X_{i}\right\|^{2}+R^{2}-$ $2 R\left\|X_{i}\right\| \cos \left(\theta\left(X_{i}\right)\right)$.

Let $\tilde{\Pi}_{s}=\left\{X_{i}, i \geq 1\right\}$ be the set including the location of the SS transmitters on a plane. The density of the SS transmitters is $\lambda_{s}$ users per unit area. We then define $\Pi_{s}=\left\{\left(X_{i}, g_{p s, i}, g_{s p, i}\right) \mid X_{i} \in \tilde{\Pi}_{s}, i \geq 1\right\}$, where $g_{p s, i}$ represents 


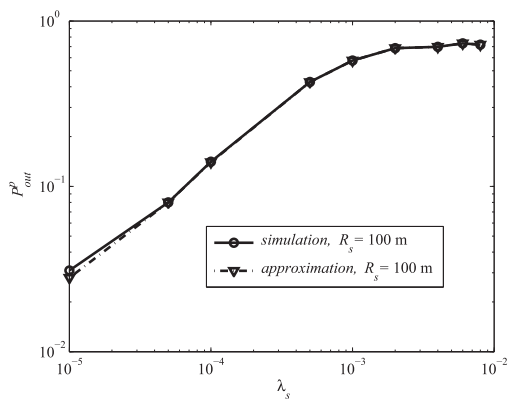

Fig. 2. PS outage probability assuming spectrum sensing (9) and equivalent miss detection (11) for Rayleigh fading. Simulation parameters are in Table 2.

the fading between the PS transmitter and the SS transmitter, and $g_{s p, i}$ is the fading between the SS transmitter and the PS receiver. Set $\Pi_{s}=\left\{\left(X_{i}, g_{p s, i}, g_{s p, i}\right) \mid X_{i} \in \tilde{\Pi}_{s}, i \geq 1\right\}$ is a homogenous PPP with density $\lambda_{s} f_{g}\left(g_{p s}\right) f_{g}\left(g_{s p}\right)$.

Let $\lambda$ be the density of those SS transmitters that experience miss detection. Set $\breve{\Pi}_{s} \subseteq \Pi_{s}$ includes $\left(X_{i}, g_{p s, i}, g_{s p, i}\right)$ where for the corresponding SS transmitter $D\left(\gamma_{p s, i}\right)=1$. We mark each point of set $\left(X_{i}, g_{p s, i}, g_{s p, i}\right) \in \Pi_{s}$ with $D\left(\gamma_{p s, i}\right)$, where $D\left(\gamma_{p s, i}\right)$ is independent among different points but depends on $\left(X_{i}, g_{p s, i}, g_{s p, i}\right)$; therefore, the set $\breve{\Pi}_{s}$ is a nonhomogeneous PPP with density $\lambda(i)=$ $\lambda_{s} f_{g}\left(g_{p s}\right) f_{g}\left(g_{s p}\right) D\left(\gamma_{p s, i}\right)$. In Appendix IV, available in the online supplemental material, we obtain the PS outage probability as the following:

$$
\begin{aligned}
& P_{\text {out }}^{p}=1-\Phi_{W}\left(\mu \frac{\gamma_{t h} R^{\alpha}}{S_{p}}\right) e^{-\lambda_{s} R^{2}\left(\frac{S_{s} \gamma_{t h}}{S_{p}}\right)^{\frac{2}{\alpha}} L} \\
& \times e^{\lambda_{s} \int_{0}^{\infty} \int_{0}^{2 \pi} \frac{u e^{-\mu\left(\frac{\omega(u, \theta)}{R_{s}^{2}}\right)^{\frac{\alpha}{2}}}}{1+\frac{S_{p} u^{\alpha}}{\gamma_{t h} S_{s} R^{\alpha}}} \mathrm{d} \theta \mathrm{d} u}
\end{aligned}
$$

Remark 3. Mathematically, this outage probability can be equal to the outage probability derived in Proposition 1 assuming the same ROC and identical miss detection probability $\delta_{e q}$, which is unknown. Our goal here is to obtain $\delta_{e q}$. To obtain $\delta_{e q}$, we can write

$$
\begin{aligned}
e^{-\lambda_{s} \delta_{e q} R^{2}\left(\frac{S_{s} \gamma_{t h}}{S_{p}}\right)^{\frac{2}{\alpha}} L}= & e^{-\lambda_{s} R^{2}\left(\frac{S_{s} \gamma_{t h}}{S_{p}}\right)^{\frac{2}{\alpha}} L} \\
& \times e^{\lambda_{s} \int_{0}^{\infty} \int_{0}^{2 \pi} \frac{u e}{1+\frac{u\left(\frac{\omega(u, \theta)}{R_{s}^{2}} \frac{S_{p}}{2}\right.}{\gamma_{t h} S_{s} R^{\alpha}}} \mathrm{d} \theta \mathrm{d} u} .
\end{aligned}
$$

Solving the above equation for $\delta_{e q}$, we have

$$
\delta_{e q}=1-\frac{\int_{0}^{\infty} \int_{0}^{2 \pi} \frac{u e^{-\mu\left(\frac{\omega(u, \theta)}{R_{s}^{2}}\right)^{\frac{\alpha}{2}}}}{1+\frac{S_{p u^{\alpha}}}{\gamma_{t h} S_{s} R^{\alpha}}} \mathrm{d} \theta \mathrm{d} u}{R^{2}\left(\frac{S_{s} \gamma_{t h}}{S_{p}}\right)^{\frac{2}{\alpha}} L} .
$$

As a result, instead of evaluating the outage probability with spectrum sensing (9), we can utilize Proposition 1 with the miss detection probability $\delta_{e q}$.

Fig. 2 illustrates that outage performance of PS in the case of actual energy detector is almost the same as what obtained assuming homogeneous ROC with equivalent miss detection $\delta_{e q}$.
Remark 4. Generally, the $i$ th miss detection probability of energy detector for given false alarm probability $\epsilon$ is obtained as $\delta_{i}=1-\epsilon^{\frac{1}{1+\gamma_{p s, i}}}$ for sensing threshold $T / N_{0} B=$ $-\ln \epsilon$ [34]. For this case, we can show that

$$
\delta_{e q}=1-\frac{\int_{0}^{\infty} \int_{0}^{2 \pi} \frac{u \mathbf{E}\left\{\epsilon^{\frac{1}{1+\frac{S_{p} \omega(u, \theta) g_{p s}}{N_{0} B}}}\right\}}{1+\frac{S_{p} u^{\alpha}}{\gamma_{t h} S_{s} R^{\alpha}}} \mathrm{d} \theta \mathrm{d} u}{R^{2}\left(\frac{S_{s} \gamma_{t h}}{S_{p}}\right)^{\frac{2}{\alpha}} L} .
$$

Note that to use simplified spectrum sensing (9), we can choose $\gamma_{0}$ by solving the following minimization:

$$
\min _{\gamma_{0}} \int_{0}^{\infty}\left(\epsilon^{\frac{1}{1+x}}-U\left(\gamma_{0}-x\right)\right)^{2} \mathrm{~d} x
$$

which is the minimizing the energy gap between real miss detection probability and miss detection probability based on approximation (9). Simple manipulation results in

$$
\min _{\gamma_{0}}\left\{\int_{0}^{\gamma_{0}}\left(\epsilon^{\frac{1}{1+x}}-1\right)^{2} \mathrm{~d} x+\int_{\gamma_{0}}^{\infty} \epsilon^{\frac{2}{1+x}} \mathrm{~d} x\right\} .
$$

Taking the differentiation with respect to $\gamma_{0}$ and letting the result equal to zero, there holds $\gamma_{0}=\frac{-\ln \epsilon}{\ln 2}-1$. Note that for small false alarm probability $\gamma_{0}$ obtained above is almost the same as sensing threshold $T / N_{0} B$. Therefore, in the small false alarm probability, sensing (9) is accurate.

This section mainly analyzed the PS outage probability in Rayleigh fading. In the next section, we elaborate new approach to approximate the primary outage with high accuracy in other fading environments.

\section{Maximum Interference Link Approximation}

In [16], [17], and [26], the outage probability in a homogenous Poisson field is approximated using a nearfield set of transmitters. Moreover, it is also shown that this approach results in tight enough lower bounds on the outage probability especially in lower outage probability regime. Therefore, one may simply use lower bounds of the outage probability in [16], [17], and [26] as an accurate approximation of the outage probability.

In this section, we derive the lower bound in [17] using a different approach and show that this lower bound is obtained by the maximum interference link approximation. We then extend this result for obtaining a tight approximation of the primary outage. We then investigate the tightness of this approximation through the analysis and simulations.

\subsection{Simple Lower Bound}

In the following, we obtain the above-mentioned lower bound based on merely the SS transmitter with the maximum imposed interference at the PS receiver. We refer to this approach as the maximum interference link approximation. We define

$$
\hat{i} \triangleq \arg \max _{\left(X_{i}, g_{s p, i}\right) \in \Pi_{s}} S_{s}\left\|X_{i}\right\|^{-\alpha} g_{s p, i},
$$




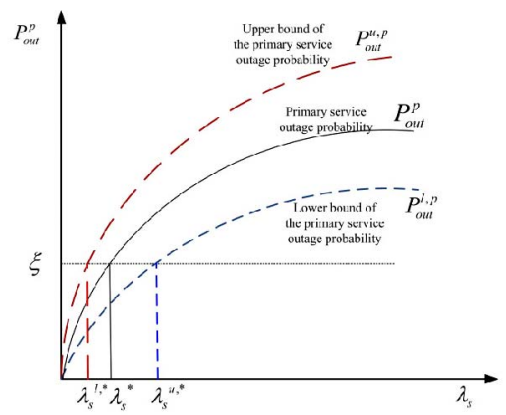

Fig. 3. The outage probability and its lower and upper bounds versus $\lambda_{s}$.

which is the secondary transmitter that imposes the maximum level of interference at the PS receiver. ${ }^{4} \mathrm{We}$ then define r.v.

$$
I_{m} \triangleq S_{s}\left\|X_{\hat{i}}\right\|^{-\alpha} g_{s p, \hat{i}}
$$

where $I_{m}$ captures the imposed interference by user $\hat{i}$. We then derive the following result.

Proposition 3. In Rayleigh fading environment, lower bound of the PS outage probability is given by (46) where it is merely derived by considering the SS transmitter $\hat{i}$, where $\hat{i}=$ $\arg \max _{\left(X_{i}, g_{s p, i}\right) \in \Pi_{s}} S_{s}\left\|X_{i}\right\|^{-\alpha} g_{s p, i}$.

Proof. See Appendix V, available in the online supplemental material.

Remark 5. It is worth mentioning that the derived lower bound in Proposition 3 is similar to the one derived in [16] for Rayleigh fading. However, comparing to [16] and [17], we derived the bound through a very easy argument. In [16] and [17], a near-field set of transmitters are considered where any individual interferer in this set may cause outage. We show that just one SS transmitter $\hat{i}$ is enough to evaluate the lower bound of the PS outage probability. Furthermore, we show that this lower bound is tight enough in the context of our system model.

In Fig. 4, the outage probability is plotted versus $\lambda_{s}{ }^{5}$ As shown, the obtained lower bound closely follows the exact PS outage probability. Consequently, one may simply adopt (46) as a performance metric that represents the PS outage probability. Using the obtained lower bound (46) may seem redundant since the exact PS outage probability is given in Proposition 1. However, this derived result is useful in more complicated scenarios such as cases with power control.

Similar to Proposition 2, one can use the result of Proposition 3 to derive the maximum SS transmitter density, $\lambda_{s}^{*}$, to satisfy the PS outage probability constraint $\xi$. Let $\lambda_{s}^{u, *}$ be an upper bound on the maximum SS transmitter density derived through letting $P_{\text {out }}^{l, p}$ equal to $\xi$. As it is depicted in

4. Note that as it is also shown in [17] employing interference cancelation in wireless ad hoc networks, by canceling the most powerful interferer, results in gaining the majority of potential performance. Nevertheless, canceling the reminder of the interferers has not have an eye-catching impact. As a consequence, we anticipate that maximum interference link approximation will successfully follow the actual outage probability.

5. Fig. 4 is partly described here; further discussions regarding the plots are presented later in this section.

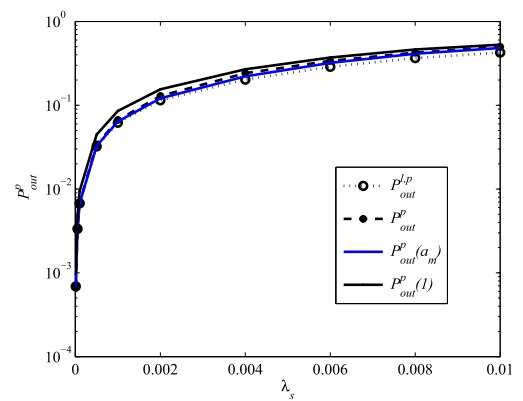

Fig. 4. The PS outage probability with different approximations for Rayleigh fading. For simulation parameters, see Table 2 in Section 8.

Fig. 3, $\lambda_{s}^{*} \leq \lambda_{s}^{u, *}$ which shows that $\lambda_{s}^{u, *}$ is an upper bound on the maximum SS transmitter density. Therefore, using $\lambda_{s}^{u, *}$ as the SS transmitter density may not satisfy the PS outage probability constraint, $\xi$, and consequently imposes unacceptable interference at the PS receiver.

Let $P_{\text {out }}^{u, p}$ be an upper bound on the PS outage probability. We may also use an upper bound on the PS outage probability, $P_{\text {out }}^{u, p}$, to derive a lower bound for the maximum SS transmitter density, $\lambda_{s}^{l, *}$. Although this approach is rather pessimistic and may sacrifice the system spectrum efficiency. Indeed, to derive an upper bound for the PS outage probability, we can use the Chernoff's bound [16], [17]. In this case, although the bound is tight, closed-form expression is not attainable. Another upper bound can be obtained utilizing Chebyshev's inequality [16]. However, the obtained upper bound in this way is usually not accurate enough which again may result in spectrum inefficiency.

To deal with the above issues, in the following, we obtain a tight approximation for the PS outage probability.

\subsection{Tight Approximation}

In this approximation, we utilize the maximum interference link approximation by introducing an extra real parameter, $a_{m}(\alpha) \geq 0$. This parameter depends on the path-loss exponent and indicates the accuracy of the proposed approximation. For the case of Rayleigh fading environment, by utilizing the obtained closed-form formula in Proposition 1, the value of this parameter is computable. Similar computation are also conducted in Proposition 4. In particular, for cases where $\gamma_{t h}$ is sufficiently small, it is shown that an accurate approximation is available if $a_{m}(\alpha) \in[0, \mathcal{C}(\alpha))$, where in such cases $\mathcal{C}(\alpha)$ is a deterministic function of $\alpha$. Indeed, for the case of $a_{m}(\alpha)=0$, the obtained approximation tends to the lower bound of the PS outage probability. If one set $a_{m}(\alpha)=\mathcal{C}(\alpha)$, the obtained upper bound based on Chebyshev's inequality proposed in [16] can be also obtained. In Section 4.3, we elaborate on the analytical details of the above explanations.

We start from $I_{m} \leq I_{\phi}$, and note that where $\gamma_{t h}$ is sufficiently small residual interference, $I_{m}^{c}$ is small enough, so that it may not usually result in an outage at the PS receiver. Therefore, one may approximate $I_{m}^{c}$ as a scaled version of $I_{m}$, i.e., $I_{m}^{c}=a_{m}(\alpha) I_{m}$, where $a_{m}(\alpha)$ is a real parameter. This is equivalent to reducing $\gamma_{t h}$ by a factor of $a_{m}(\alpha)+1$ in evaluating the lower bound of the outage since 


$$
\mathbf{P}\left\{I_{\phi} \geq z\right\}=\mathbf{P}\left\{I_{m} \geq \frac{z}{a_{m}(\alpha)+1}\right\} .
$$

Here, parameter $a_{m}(\alpha)+1$ compensates the impact of the aggregated interference $I_{m}^{c}$ on the PS outage probability in Proposition 3. Optimal $a_{m}(\alpha)$ results in accurate evaluation of the outage probability through the lower bound.

In cases, where the path-loss exponent is high, i.e., $3 \lesssim$ $\alpha<6$ one can intuitively claim that $I_{m}^{c}=a_{m}(\alpha) I_{m}$. In fact, in this case, the secondary transmitters implied aggregate interference at the primary receiver does not have a serious role in experiencing outage by the primary receiver. In the other words, due to high path-loss exponent, it is most likely that the received power level at the primary receiver is already too weak; thus, the PS is in outage even without considering the SS implied interference. Consequently, in such cases, considering a small real value for parameter $a_{m}(\alpha)$ results in a reasonable approximation.

For the cases, where $2<\alpha \lesssim 3$, the dominant source of the PS outage is the aggregated interference implied by the SSs. In this case, the primary receiver experiences outage if strong enough interference is implied by the SS transmitter $\hat{i}$. Since distance-dependent path-loss attenuation is actually small, the outage may be also experienced in result of interference implied by the other secondary transmitters. Therefore, corresponding to $2<\alpha \approx 3$, considering a positive small real value for $a_{m}(\alpha)$ provides a better approximation comparing to $a_{m}(\alpha)$ to zero.

Since the pdf of r.v. $I_{m}$ is known, ignoring the noise contribution, we can obtain an approximation for the PS outage probability as

$$
\begin{aligned}
P_{\text {out }}^{p} & \approx \mathbf{E}\left[\mathbf{P}\left\{\left(1+a_{m}(\alpha)\right) I_{m} \geq \frac{S_{p} R^{-\alpha} g_{p}}{\gamma_{t h}} \mid g_{p}\right\}\right] \\
& =1-\mathbf{E}_{g}\left[e^{-\pi \lambda\left(\frac{\left(1+a_{m}(\alpha)\right) S_{s} \gamma_{t h}}{\mu S_{p}}\right)^{\frac{2}{\alpha}} R^{2} g_{p}^{-\frac{2}{\alpha}} \Gamma\left(1+\frac{2}{\alpha}\right)}\right] \\
& \triangleq P_{\text {out }}^{p}\left(a_{m}(\alpha)\right) .
\end{aligned}
$$

To estimate parameter $a_{m}$ and ignoring the impact of the additive noise, we can use the result of Proposition 1 together with (14) and write

$$
\mathbf{E}_{g}\left[e^{-\pi \lambda\left(\frac{\left(1+a_{m}(\alpha)\right) S_{S} \gamma_{t h}}{\mu S_{p}}\right)^{\frac{2}{\alpha}} R^{2} g_{p}^{-\frac{2}{\alpha}} \Gamma\left(1+\frac{2}{\alpha}\right)}\right]=e^{-\lambda R^{2}\left(\frac{S_{s} \gamma_{t h}}{S_{p}}\right)^{\frac{2}{\alpha}} L} .
$$

Using first order Taylor extension of $e^{-x}$ as $e^{-x} \approx 1-x$, an approximated value of parameter $a_{m}(\alpha)$ is

$$
a_{m}(\alpha) \approx 1-\left(\frac{1-e^{-\lambda R^{2}\left(\frac{S_{s} \gamma_{t h}}{S_{p}}\right)^{\frac{2}{\alpha}} L}}{\pi \lambda\left(\frac{S_{s} \gamma_{t h}}{S_{p}}\right)^{\frac{2}{\alpha}} R^{2} \Gamma\left(1-\frac{2}{\alpha}\right) \Gamma\left(1+\frac{2}{\alpha}\right)}\right)^{\frac{\alpha}{2}},
$$

where we set $\mathbf{E}\left[g_{s p}^{-\frac{2}{\alpha}}\right]=\mu^{\frac{2}{\alpha}} \Gamma\left(1-\frac{2}{\alpha}\right)$. The above results are presented in the following proposition.

Proposition 4. An approximation of the PS outage probability in the Rayleigh fading environment is $P_{\text {out }}^{p}\left(a_{m}(\alpha)\right)$ in (14), where parameter $a_{m}(\alpha)$ is obtained adaptively based on (16).

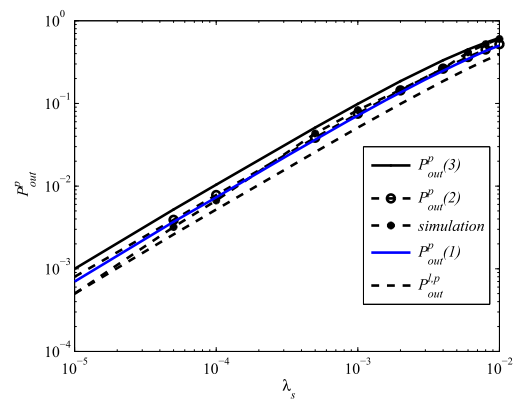

Fig. 5. The PS outage probability with different approximations in lognormal shadowing environment for $m_{s}=m_{p}=1, v_{s}=v_{p}=4$. Simulation parameters are in Table 2.

In Fig. $4,(14)$ is plotted considering the obtained $a_{m}(4)$, $\alpha=4$, from (16). As it is seen, $P_{\text {out }}^{p}\left(a_{m}(4)\right)$ closely follows $P_{\text {out }}^{p}$. However, in practice, evaluating $a_{m}(4)$ may be difficult due to the lack of the closed-form expression of the outage probability especially in more complicated scenarios and/ or considering other fading environments. We further observe that by setting $a_{m}(4)=1, P_{\text {out }}^{p}(1)$ also provides a tight approximation of the PS outage probability as it is shown in Fig. 4.

Example 1 (Log-normal shadowing). Here, we obtain the lower bound in (45) for the case of log-normal shadowing. Let $g_{s p}$ and $g_{p}$ be log-normal r.v.s with parameters $\left(m_{s}, v_{s}\right)$, and $\left(m_{p}, v_{p}\right)$, respectively. Log-normal r.v. $X$ with parameter $(m, v)$ has the following pdf [30]:

$$
f_{X}(x)=\frac{1}{x \sqrt{2 \pi \sigma^{2}}} e^{-\frac{(\log (x)-\eta)^{2}}{2 \sigma^{2}}} 1_{x>0},
$$

where $\eta$ and $\sigma$ are obtained through the following equalities:

$$
\eta=\log \left(\frac{m^{2}}{\sqrt{v+m^{2}}}\right), \sigma=\sqrt{\log \left(\frac{v}{1+m^{2}}\right)} .
$$

Straightforward derivations yields $\mathbf{E}\left[g_{s p}^{\frac{2}{\alpha}}\right]=e^{\frac{2 \eta}{\alpha}+\frac{2 \sigma_{s}^{2}}{\alpha^{2}}}$. Using (45), a lower bound for the PS outage probability is then obtained as

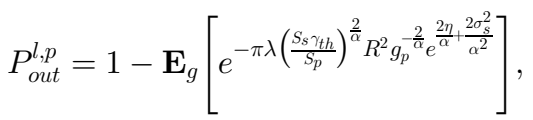

consequently, a tight approximation for the PS outage probability using the result of Remark 2 is obtained as

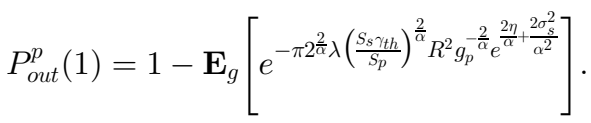

Fig. 5 illustrates the PS outage probability in lognormal shadowing environment. In this figure, we also illustrate the lower bound of the PS outage probability, $P_{o u t}^{l, p}$. As it is seen in this case, the presented lower bound is also tight; however, the gap is greater than that of the case of Rayleigh fading. Moreover, in Fig. 5, $P_{\text {out }}^{p}\left(a_{m}(4)\right)$ for $a_{m}(4)=1,2,3$ is also added. As it is observed in this case, $P_{\text {out }}^{p}(1)$ also provides a very tight approximation of the PS outage probability. 
TABLE 1

Values of $\mathcal{C}(\alpha)$

\begin{tabular}{c||c}
\hline$\alpha$ & $\mathcal{C}(\alpha)$ \\
\hline \hline 2.0 & 0 \\
2.5 & 0.0686 \\
3.0 & 0.2823 \\
3.5 & 0.4960 \\
4.0 & 0.7097 \\
4.5 & 0.9234 \\
5.0 & 1.1371 \\
\hline
\end{tabular}

\subsection{Discussion}

Consider the obtained lower bound in (45). An approximation of the PS outage probability is obtained by replacing $\lambda_{s}$ with a scaled version of the transmitter density $2^{\frac{1+a_{m}(\alpha)}{\alpha}} \lambda_{s}$. For a given $a_{m}$, adopting the asymptotic expansion in [16], the following is obtained for the case that $\gamma_{t h}$ is small enough

$$
\begin{aligned}
P_{o u t}^{l, p} & =\pi \lambda_{s} \delta R^{2} \mathbf{E}\left[g_{s p}^{\frac{2}{\alpha}}\right] \mathbf{E}\left[g_{p}^{\frac{-2}{\alpha}}\right] \gamma_{t h}^{\frac{2}{\alpha}}+\mathcal{O}\left(\gamma_{t h}^{\frac{4}{\alpha}}\right), \\
P_{o u t}^{u, p} & =\frac{\alpha}{\alpha-1} \pi \lambda_{s} \delta R^{2} \mathbf{E}\left[g_{s p}^{\frac{2}{\alpha}}\right] \mathbf{E}\left[g_{p}^{\frac{-2}{\alpha}}\right] \gamma_{t h}^{\frac{2}{\alpha}}+\mathcal{O}\left(\gamma_{t h}^{\frac{4}{\alpha}}\right), \\
P_{\text {out }}^{p}\left(a_{m}(\alpha)\right) & =\pi 2^{\frac{1+a_{m}(\alpha)}{\alpha}} \lambda_{s} \delta R^{2} \mathbf{E}\left[g_{s p}^{\frac{2}{\alpha}}\right] \mathbf{E}\left[g_{p}^{\frac{-2}{\alpha}}\right] \gamma_{t h}^{\frac{2}{\alpha}}+\mathcal{O}\left(\gamma_{t h}^{\frac{4}{\alpha}}\right) .
\end{aligned}
$$

If $a_{m}(\alpha)=0$, the lower bound and the proposed approximation are asymptotically equivalent. However, for obtaining a better approximation comparing to the upper bound obtained in [16], it is necessary to have

$$
0 \leq a_{m}(\alpha) \leq\left(\alpha \log _{2}\left(\frac{\alpha}{\alpha-1}\right)-1\right)^{+} \triangleq \mathcal{C}(\alpha) .
$$

In Table $1, \mathcal{C}(\alpha)$ is given for different values of $\alpha$. Note that $\mathcal{C}(\alpha)$ provides an upper bound on the value of $a_{m}(\alpha)$ for the cases where $\gamma_{t h}$ is small enough. Later, in Section 8, it is further observed that even selecting a bit larger values for $a_{m}(\alpha)$, may not result in a meaningful increase in the gap between the actual simulated PS outage probability, and the obtained approximation. The above explanations are presented in Remark 6

Remark 6. For a spectrum sharing system with the SS density of $\lambda_{s}$ and path-loss exponent of $\alpha$, substituting $\lambda_{s}$ in (46) with $2^{\frac{1+a_{m}(\alpha)}{\alpha}} \lambda_{s}$ results in a very close approximation of the actual outage probability, where $a_{m}(\alpha) \in[0, \mathcal{C}(\alpha))$.

In Fig. 6, we present the impact of the path-loss exponent, $\alpha$, on the PS outage probability, where results of various approximations are also provided. The simulation parameters and scenario are presented in Section 8. In this simulation, the thermal noise effect is also considered. As it is observed, for small and moderate values of $\alpha$, i.e., $2 \leq \alpha<4$, the proposed approximation with parameter $a_{m}(\alpha)=\mathcal{C}(\alpha) / 3$ outperforms the others. For the case of $\alpha>4$, the approximations perform similar to each other.

Fig. 6 also indicates that for $2 \leq \alpha \leq 3.5$, the distancedependence path-loss attenuation has a positive impact on the PS outage performance. This is mainly due to the fact that the received interference at the primary receiver is reduced since higher path loss is experienced in the link between the secondary transmitter and the primary

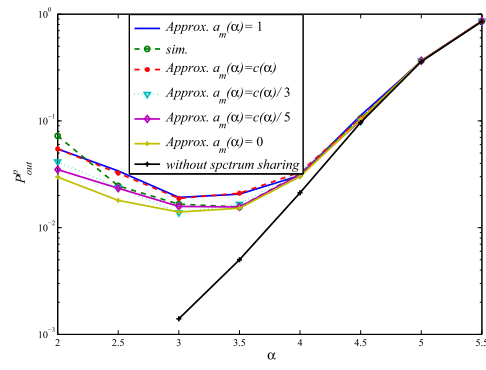

Fig. 6. The PS outage probability versus the path-loss exponent with various approximations for Rayleigh fading. Simulation parameters are presented in Table 2 and in Section 8.

receiver. It is also seen that increasing $\alpha$ beyond 3.5 dramatically increases the PS outage probability. In this case, the received power corresponding to the primary transmitter at the primary receiver is most likely too weak and the PS is in the outage, even without considering the imposed interference.

\section{ImPACT OF SS POWER CONTROL}

In some circumstances, although a secondary transmitter detects the spectrum state as idle, the channel between this secondary transmitter and its corresponding secondary receiver may experience deep fading. In such cases, accessing to the spectrum has no benefit for the SS user. Furthermore, such transmission may impose interference at the PS receiver, for example, in miss detection event.

Therefore, a power control strategy based on the channel condition between the SS transmitter and receiver might improve the SS power usage as well as the PS outage performance.

In wireless communications, power control is usually adopted to overcome the fading effects of wireless channel with the objective of increasing spectral efficiency. In power control, the transmitter usually calculates the appropriate transmission power based on the channel condition between itself and the peer receiver either to maximize its power usage, or to optimize other objectives such as throughput.

Various power control strategies with different objectives are proposed in the related literature, see, for example, [30]. Here, to show the impact of power control strategy on the performance of the spectrum sharing, we study the impact of truncated channel inversion power control on the performance of spectrum sharing. Truncated channel inversion power control is shown to be efficient, and at the same time simply implementable [30].

In [23], the authors briefly mentioned the effect of the linear role of the statistics of the adopted power control mechanism on the cumulants of the aggregate interference at the PS receiver. In this paper, we elaborate on the impacts of the power control on the PS outage probability. Here, we obtain a tight approximation of the PS outage probability based on the maximum link approximation. In Section 8, we show that power control mechanism is able to reduce the PS outage probability. Moreover, later, in Section 7, we show that the SS power control also improves the SS mean spatial throughput.

Here, we examine the effect of truncated channel inversion power control on the PS outage performance. 
Similar to the argument in Section 3, here we define $\tilde{\Pi}_{s}=$ $\left\{X_{i}, i \geq 1\right\}$ as a set that includes the locations of the SS transmitters. Set $\tilde{\Pi}_{s}$ is homogenous PPP with density $\lambda_{s}$. Each point $X_{i} \in \tilde{\Pi}_{s}$ is marked by the fading pair $\left(g_{s s, i}, g_{s p, i}\right)$ to produce a new set $\Pi_{s}=\left\{\left(X_{i}, g_{s s, i}, g_{s p, i}\right) \mid X_{i} \in \tilde{\Pi}_{s}, i \geq 1\right\}$. The marking corresponding to each point is independent from the others due to three following reasons: 1) the independency of the fading of the channel between different SS transmitters located at $X_{i}, i=1,2, \ldots$, and the PS receiver located at the origin, i.e., $g_{s p, i}, 2$ ) the independency of the fading of the channel between the SS transmitter and its receiver located at $X_{i}, i=1,2, \ldots$, i.e., $g_{s s, i}$, and 3 ) the independency between $g_{s p, i}$ and $g_{s s, i}$ for each point $X_{i}, i=$ $1,2, \ldots$ Therefore, it is straightforward to show that $\Pi_{s}$ is a homogenous PPP with density $\lambda_{s} f_{g}\left(g_{s s}\right) f_{g}\left(g_{s p}\right)$. We define $\breve{\Pi}_{s}=\left\{\left(X_{i}, g_{s s, i}, g_{s p, i}\right) \mid X_{i} \in \tilde{\Pi}_{s}, D_{i}=1, i \geq 1\right\}$ as a set including the location of the SS transmitters with miss detection. The Coloring Theorem [7] shows that $\breve{\Pi}_{s}$ is a homogenous PPP.

In truncated channel inversion, the SS receiver $i$ feedbacks $g_{s s, i}$ to the corresponding transmitter; then, the transmitter adjusts its power based on

$$
S_{i}=\frac{T}{y_{i}^{-\alpha} g_{s s, i}} 1_{g_{s s, i}>\frac{T}{S_{s}} y_{i}^{\alpha}},
$$

where $T$ is a system parameter indicating the minimum required received power strength at the SS receiver $i$. In this strategy, the SS transmitter $i$ adjusts its transmit power, subject to its maximum transmission power constraint, so that the received power at its receiver is kept equal to $T$. In (19), $y_{i}$ is the distance between receiver $i$ and the secondary transmitter, where for brevity we assume $y_{i}=y, \forall i$. The considered truncated channel inversion power control may be considered as an combination of the channel inversion power control and threshold based power control mechanisms considered in [16]. In the following study, we derive enough tight approximation of the PS outage probability.

The density of transmitters belonging to set $\breve{\Pi}_{s}$ is $\lambda=\delta \lambda_{s} e^{-\mu \frac{T}{S_{s}} y^{\alpha}}$. In this case, a closed-form expression of the PS outage probability may not be accessible. Thus, for $W \ll I_{\phi}$, utilizing the proposed analysis in Section 4, we derive a tight approximation for the PS outage probability in Appendix VII, available in the online supplemental material:

$$
\begin{aligned}
P_{\text {out }}^{p}\left(a_{m}(\alpha)\right)= & 1-\mathbf{E}\left[\operatorname { e x p } \left\{-\pi \delta\left(1+a_{m}(\alpha)\right)^{\frac{2}{\alpha}} \lambda_{s} e^{-2 \mu \frac{T}{S_{s}} y^{\alpha}}\right.\right. \\
& \left.\left.\times \int_{0}^{\infty} \frac{r e^{-\frac{\mu S_{p} g_{p}}{S_{s} \gamma_{t h}}\left(\frac{r}{R}\right)^{\alpha}}}{1+\mu \frac{\gamma_{t h} T}{S_{p} g_{p}}\left(\frac{y R}{r}\right)^{\alpha}} \mathrm{d} r\right\}\right]
\end{aligned}
$$

Simulation results in Section 8 show that $P_{\text {out }}^{p}(1)$ is a tight approximation of the PS outage probability.

\section{Cooperative Spectrum Sensing}

Here, we examine the developed framework to the cooperative sensing scenario. In cooperative sensing, the SS transmitters/sensors share their sensing knowledge with each other to improve the sensing performance. Cooperative spectrum sensing schemes are categorized into two following categories: centralized and decentralized. In the centralized cooperative spectrum sensing, all SS transmitters/sensors send their spectrum sensing data to a central decision making entity that is called data fusion center to evaluate the spectrum availability.

In the decentralized cooperative spectrum sensing, SS transmitters share their sensing information with the adjacent SS transmitters. Based on the aggregated sensing information, the spectrum availability is evaluated. Here, we analyze both centralized and decentralized cooperative spectrum sensing schemes. We utilize models in Section 3.

\subsection{Centralized Cooperative Spectrum Sensing}

The fusion center utilizes a rule to evaluate the spectrum state based on the sensed data. A survey on the spectrum sensing methods can be found in, for example, [35]. Here, for instance, we focus only on AND rule as well as voting rule.

Based on the AND rule, if all received sensing data from the SS transmitters indicate idle state, the fusion center evaluates the spectrum as idle.

In the fusion center, we define $D=\sum_{X_{i} \in \breve{\Pi}_{s}} \mathbf{1}_{\left\{D_{i}=1\right\}}$ based on AND rule if $D=N\left(\breve{\Pi}_{s}\right)\left(D<N\left(\breve{\Pi}_{s}\right)\right)$; thus, the spectrum is identified as idle (busy) where $N(A)$ denotes the number of the points fall in set $A \subset \mathbb{R}^{2}$. The following proposition obtains the density of the SS transmitters with miss detection, $\lambda$, in the fusion center based on the AND rule.

Proposition 5. Consider a centralized cooperative spectrum sensing based on AND rule with large enough number of sensed data, where the individual miss detection probability for each secondary transmitter is $0<\delta<1$. In such system, the density of the SS transmitters with miss detection is zero.

Proof. See Appendix VIII, available in the online supplemental material.

Proposition 5 shows that although miss detection is probable at each SS transmitter, utilizing centralized cooperative spectrum sensing, the SS network acts such that miss detection is not probable. Note that accurate idle state estimation with centralized cooperative spectrum sensing is achieved with the cost of imposing a huge signaling overhead. In practice, however, using only one data fusion center may not be possible; therefore, considering multiple data fusion centers is more appropriate.

In practice, AND rule lies on the pessimistic side from the SS performance point of view. Indeed, although the actual density of the secondary transmitters with miss detection experience approaches to zero, it may reversely cause the related false alarm probability tends 1 . As a result, many of the secondary transmitters may experience false alarm almost all the time. Consequently, the OSA may fail to gain reasonable spectral efficiency.

To tackle this issue, assume the data fusion center identifies the spectrum as an idle through $M$-out-of- $N\left(\breve{\Pi}_{s}\right)$ rule; if it receives more than $M$ availability indicators, where $0 \leq M \leq N\left(\breve{\Pi}_{s}\right)$, the spectrum status is evaluated as idle; otherwise, it is evaluated as busy. In the following, we briefly study the impact of this rule on the PS outage 
probability. For mathematical tractability, we assume that the secondary network is geographically located into a disk centered at the origin with radius $d \gg 1, \mathcal{O}_{d}(o)$. Tending $d \rightarrow \infty$, the desired result is then obtained.

Now, we consider a unit area region, $A \subset \mathcal{O}_{d}(o)$. The density of the secondary nodes with miss detection is given by $\lambda=\lambda_{s} \int_{\mathcal{O}_{d}(o)} \mathbf{1}_{\{x \in A\}} \mathbf{P}\left\{\sum_{X_{i} \in \breve{\Pi}_{s} \cap \mathcal{O}_{d}(o)} \mathbf{1}_{\left\{D_{i}=1\right\}}>M\right\} \mathrm{d} x$. For Homogenous PPP $\breve{\Pi}_{s}$, it is verified that

$$
\begin{aligned}
& \mathbf{P}\left\{\sum_{X_{i} \in \breve{\Pi}_{s} \bigcap_{\mathcal{O}_{d}(o)}} \mathbf{1}_{\left\{D_{i}=1\right\}}>M\right\} \\
& =1-\mathbf{P}\left\{\sum_{X_{i} \in \breve{\Pi}_{s} \bigcap_{\mathcal{O}_{d}(o)}} \mathbf{1}_{\left\{D_{i}=1\right\}} \leq M\right\} \\
& =1-\mathbf{P}\left\{\bigcup_{m=0}^{M} \sum_{X_{i} \in \breve{\Pi}_{s} \cap \mathcal{O}_{d}(o)} \mathbf{1}_{\left\{D_{i}=1\right\}}=m\right\} \\
& =1-e^{-\lambda_{s} \delta \pi d^{2}} \sum_{m=0}^{M} \frac{\left(\lambda_{s} \delta \pi d^{2}\right)^{m}}{m !} .
\end{aligned}
$$

Consequently, $\lambda$ is

$$
\lambda=\lambda_{s}\left(1-e^{-\lambda_{s} \delta \pi d^{2}} \sum_{m=0}^{M} \frac{\left(\lambda_{s} \delta \pi d^{2}\right)^{m}}{m !}\right) .
$$

If $d \rightarrow \infty$, considering $\lim _{x \rightarrow \infty} \sum_{m} x^{m} e^{-x}=0$, (22) reduces to $\lambda_{s}$. By increasing $d$ to infinity for any finite value of $M$, the fusion center may receive, with high probability, larger than $M$ indicators standing for the availability of the spectrum. In fact, secondary transmitters located far from the primary transmitter usually experience miss detection. As a result, if the network covers $\mathbb{R}^{2}$, for given $M<N\left(\breve{\Pi}_{s}\right)$ the $M$-out-of- $N\left(\breve{\Pi}_{s}\right)$-rule does not provide any benefits on reducing the density of the secondary transmitters with miss detection experience.

In practical cases, where the network is in fact located in a region with finite area, the equivalent miss detection probability observed in the secondary network is $\delta_{e q}=$ $1-e^{-\lambda_{s} \delta \pi d^{2}} \sum_{m=0}^{M} \frac{\left(\lambda_{s} \delta \pi d^{2}\right)^{m}}{m !}$. Parameter $\delta_{e q}$ can be smaller or higher than $\delta$ by choosing proper value for $M$ corresponding to given $\lambda_{s}$ and $d$. The above results are also verified through the simulations in Section 8 . In this case, a tradeoff between the desirable false alarm probability, i.e., the SS spectral efficiency, and the equivalent miss detection probability, i.e., the PS outage probability, can be established, but it is out of the scope of this paper.

For the voting rule discussed above, we also present the following remarks:

Remark 7. Note that it is easy to verify that by letting $M \rightarrow$ $N\left(\breve{\Pi}_{s}\right)$ and $d \rightarrow \infty$, the same result as of Proposition 5 is obtained.

Remark 8. In Section 8, through the simulations, we observe that the PS outage probability with $M$-out-of- $N\left(\breve{\Pi}_{s}\right)$ rule exposes two following distinct behaviors. For secondary transmitter density, $\lambda_{s}$, smaller than critical density,
$\lambda_{s}^{*}(M)$, the SS acts similar to a network with miss detection probability near to zero. Nevertheless, in the case that $\lambda_{s}>\lambda_{s}^{*}(M)$ the secondary network acts similar to a network with miss detection probability one. More details can be found in Fig. 11. We can assume that the PS outage probability behaves similar to $U\left(\lambda_{s}-\lambda_{s}^{*}(M)\right)$ where this approximation is tight for $M \gg 1$. For the case of Rayleigh fading, we can evaluate that an estimate $\lambda_{s}^{*}(M)$ will be the solution of the following nonlinear equation (see Appendix IX, available in the online supplemental material):

$$
\begin{aligned}
& \lambda_{s}^{*}(M) e^{-\frac{\lambda_{s}^{*}(M) \delta \delta d^{2}}{M+2}}\left(1+\sum_{m=1}^{M^{*}-M} \frac{\left(\lambda_{s}^{*}(M) \delta \pi d^{2}\right)^{m}}{\prod_{n=1}^{m}(M+n+1)}\right)^{\frac{1}{M+2}} \\
& =(2 \pi(M+1))^{\frac{1}{2 M+4}}\left(\frac{M+1}{e \delta \pi d^{2}}\right)^{\frac{M+1}{M+2}}\left(\frac{\ln \left(2 \Phi_{W}(\Omega)\right)}{\Xi}\right)^{\frac{1}{M+2}} .
\end{aligned}
$$

For $M^{*}=20$ and simulations parameters of Section 8 , we find that $\lambda_{s}^{*}(1,000)=3.17 \times 10^{-5}$ and $\lambda_{s}^{*}(10,000)=$ 0.0002063 which are very close to what we obtained through simulations in Fig. 11.

\subsection{Decentralized Cooperative Spectrum Sensing}

In decentralized spectrum sensing, each SS transmitter shares its sensed data with all transmitters located in its neighborhood. Here, we again take AND rule as an example, in which in transmitter $i$, the spectrum is evaluated as idle if all received sensed data as well as the sensed data in the transmitter $i$ evaluate the spectrum status as idle. We assume that each SS transmitter can share its sensed information inside a circular neighborhood with radius $R_{c}$. We refer to $R_{c}$ as the cooperation radius. Thus, all the SS transmitters that are located inside this circle are able to receive the sensed data.

In [23], authors employ the decentralized cooperative spectrum sensing through amplify-and-forward relaying of the out-of-band bacon signal, broadcasted by the primary receiver. Each secondary transmitter broadcasts the received bacon signal to the other transmitters falling in a circular neighborhood with radius $R_{s}$. However, in their analysis, the spatial density of transmitters with miss detection in [23] needs numerical evaluation of double integrals. Furthermore, they only presented their results for the case where $R_{c}$ is small enough.

Our analysis, on the other hand, is general enough to cover any range of $R_{c}$. Moreover, we derive a closed-form expression of the spatial density of secondary transmitters with miss detection, and mathematically proved that for large enough $\lambda_{s}$ employing decentralized cooperative spectrum sensing the obtained PS outage probability is dramatically reduced comparing to the cases without cooperation. The main result on decentralized spectrum sensing is presented in the following proposition:

Proposition 6. For a decentralized cooperative spectrum sensing based on AND rule with cooperation radius of $R_{c}$, the density of the SS transmitter with miss detection event is obtained as

$$
\lambda=\delta \lambda_{s} e^{-(1-\delta) \lambda_{s} \pi R_{c}^{2}} .
$$


Proof. See Appendix $X$, available in the online supplemental material.

Remark 9. It is seen that a decentralized cooperative spectrum sensing based on individual spectrum sensing procedures with detection probability $\delta$ is equivalent to noncooperative spectrum sensing with an individual spectrum sensing procedure having miss detection probability of $\delta_{e q}=\delta e^{-(1-\delta) \lambda_{s} \pi R_{c}^{2}}<\delta$. Note that if $R_{c} \rightarrow \infty$, then $\lambda \rightarrow 0$, and the negative impact of the SS on the PS is completely vanished. In this case, the centralized and decentralized cooperative spectrum sensing act similarly.

Remark 10. As we also show by simulation in Section 8 , due to increasing $\lambda_{s}$, two different behaviors are demonstrated by the PS outage probability. On one hand, if the density of the SS transmitters is small enough, by increasing $\lambda_{s}$ from zero to $\tilde{\lambda}_{s}$, the outage probability of the PS is increased. In this region, the PS outage probability follows the same pattern as was obtained in Section 3. On the other hand, for $\lambda_{s}>\tilde{\lambda}_{s}$, the PS outage probability decreases by increasing $\lambda_{s}$. In the case that $\lambda_{s}<\tilde{\lambda}_{s}$, the number of SS nodes are not enough to establish an effective cooperative spectrum sensing. Therefore, cooperative spectrum sensing does not make enough improvement to decrease the PS outage probability. However, in the case that $\lambda_{s}>\tilde{\lambda}_{s}$, cooperative spectrum sensing effectively reduces the PS outage probability. This is because of the fact that each SS transmitter is able to share its sensing knowledge with the other transmitters.

It is easy to verify that the PS outage probability for the case of Rayleigh fading

$$
P_{\text {out }}^{p}=1-\Phi_{W}\left(\mu \frac{\gamma_{t h} R^{\alpha}}{S_{p}}\right) e^{-\lambda_{s} \delta e^{-(1-\delta) \lambda_{s} \pi R_{c}^{2}} R^{2}\left(\frac{S_{s} \gamma_{t h}}{S_{p}}\right)^{\frac{2}{\alpha}} L},
$$

is a convex function respect to $\lambda_{s}$. The amount of $\tilde{\lambda_{s}}$ can then be obtained by taking differentiation with respect to $\lambda_{s}$ and letting the result equal to zero as

$$
\tilde{\lambda_{s}}=\frac{e^{-(1-\delta) \tilde{\lambda}_{s} \pi R_{c}^{2}}}{(1-\delta) \pi R_{c}^{2}} \approx \frac{1}{2(1-\delta) \pi R_{c}^{2}} .
$$

Remark 11. For small enough values of $\lambda_{s}$, the PS outage probability with and without centralized cooperative spectrum sensing performs almost identically. Therefore, it is necessary to find the critical spatial density $\lambda_{s}^{c r}(\Delta)$ that for $\lambda_{s}>\lambda_{s}^{c r}(\Delta)$ centralized cooperative spectrum sensing benefits the SS in reducing the PS outage probability. Here, $\Delta \ll 1$ is the gap between the PS outage probability with and without centralized cooperation. For the case of Rayleigh fading, Proposition 1 holds

$$
\begin{aligned}
& e^{-\lambda_{s}^{c r}(\Delta) \delta R^{2}\left(\frac{S_{s} \gamma_{t h}}{S_{p}}\right)^{\frac{2}{\alpha}} L}-e^{-\lambda_{s}^{c r}(\Delta) \delta_{e q} R^{2}\left(\frac{S_{s} \gamma_{t h}}{S_{p}}\right)^{\frac{2}{\alpha}} L} \\
& =\frac{\Delta}{\Phi_{W}\left(\mu \frac{\gamma_{t h} R^{\alpha}}{S_{p}}\right)},
\end{aligned}
$$

where $\delta_{e q}=\delta e^{-(1-\delta) \lambda_{s} \pi R_{c}^{2}}$. The left-hand side of (25) may be simplified as the following:

$$
\begin{aligned}
& e^{-\lambda_{s}^{c r}(\Delta) \delta R^{2}\left(\frac{S_{s} \gamma_{t h}}{S_{p}}\right)^{\frac{2}{\alpha}} L}-e^{-\lambda_{s}^{c r}(\Delta) \delta_{e q} R^{2}\left(\frac{S_{s} \gamma_{t h}}{S_{p}}\right)^{\frac{2}{\alpha}} L} \\
& \quad \approx \lambda_{s}^{c r}(\Delta) R^{2}\left(\frac{S_{s} \gamma_{t h}}{S_{p}}\right)^{\frac{2}{\alpha}} L\left(\delta-\delta_{e q}\right) \\
& =\lambda_{s}^{c r}(\Delta) R^{2}\left(\frac{S_{s} \gamma_{t h}}{S_{p}}\right)^{\frac{2}{\alpha}} L \delta\left(1-e^{-(1-\delta) \lambda_{s}^{c r}(\Delta) \pi R_{c}^{2}}\right) \\
& \approx\left(\lambda_{s}^{c r}(\Delta)\right)^{2} \delta(1-\delta) \pi R_{c}^{2} R^{2}\left(\frac{S_{s} \gamma_{t h}}{S_{p}}\right)^{\frac{2}{\alpha}} L .
\end{aligned}
$$

An approximation of $\lambda_{s}^{c r}(\Delta)$ is then obtained as follows:

$$
\lambda_{s}^{c r}(\Delta) \approx \frac{\left(\frac{S_{s} \gamma_{t h}}{S_{p}}\right)^{\frac{-1}{\alpha}}}{R R_{c}} \sqrt{\frac{\Delta}{\delta(1-\delta) \pi L \Phi_{W}\left(\mu \frac{\gamma_{t h} R^{\alpha}}{S_{p}}\right)}} .
$$

This indicates that if $\lambda_{s}<\lambda_{s}^{c r}(\Delta)$, the SS does not need to employ decentralized cooperation.

\section{SS Performance}

So far, we study the outage probability of the PS and examine the impact of the power control mechanism and cooperative spectrum sensing on it. In this section, we briefly study the performance of the SS. Here, we are interested in knowing the impact of PS outage probability on the SS performance. We also want to know the effect of power control mechanism and cooperative spectrum sensing on the SS performance. Due to space limitation, we briefly study the mean spatial throughput of the SS. For other potential performance measures, refer to [16] and [12] and references therein.

Note that the SS is able to gain throughput not only in correctly detected spectrum holes, but also during miss detection experiences. The SS performance in miss detection depends among other things on mutual interference among secondary nodes as well as the interference imposed at the secondary receivers due to PS operations. The latter can complicate our analysis; thus, for brevity, we only focus in examining the performance of the SS in correctly detected spectrum holes. Notice that secondary nodes far from primary transmitters can highly gain throughput in miss detection experiences; thus, the presented analysis here only demonstrated a lower bound of the available SS spatial throughput.

First of all, we focus on the system model considered in Section 3.2, a secondary system without power control mechanism and cooperation. Utilizing results of Proposition 2, we are able to compute maximum secondary transmitter density, $\lambda_{s}^{*}$, for given outage probability constraint $\xi$ and miss detection probability $\delta$. Note that based on the obtained results in Sections 3.3 and 4, we are able to compute $\lambda_{s}^{*}$ for more complicated situations. We define set $\Lambda_{s}^{*}$ as $\Lambda_{s}^{*}=\left[0, \lambda_{s}^{*}\right]$. Therefore, given $\lambda_{s} \in \Lambda_{s}^{*}$, the secondary transmitters with truly detected spectrum holes form a homogenous PPP with density $(1-\epsilon) \lambda_{s}$. We define $\mathrm{SINR}_{s, i}$ as the SINR at the $i$ th receiver. $\mathcal{T}\left(y,(1-\epsilon) \lambda_{s}^{*}\right)$ is then defined as the mean spatial throughput of node $i$ that is obtained as follows [12], [36]:

$$
\mathbf{E} \log \left(1+\operatorname{SINR}_{s, i}\right)=\int_{0}^{\infty} \mathbf{P}\left\{\log \left(1+\operatorname{SINR}_{s, i}\right)>r\right\} \mathrm{d} r .
$$


Similar to what we have derived for the PS outage probability can be obtained for evaluating $\mathbf{P}\left\{\log \left(1+\operatorname{SINR}_{s, i}\right)>r\right\}$. For a given set $B \in \mathbb{R}^{2}$ with $|B|=1$ and noting Palm distribution, the spatial throughput is then obtained as [12]

$$
\begin{aligned}
G & =\frac{1}{|B|} \mathbf{E} \sum_{i \in B} e_{i} \log \left(1+\operatorname{SINR}_{s, i}\right) \\
& =(1-\epsilon) \lambda_{s} \int_{x \in B} \mathbf{E} \log \left(1+\operatorname{SINR}_{s, x}\right) \mathrm{d} x \\
& =(1-\epsilon) \lambda_{s} \int_{0}^{\infty} \mathbf{P}\left\{\operatorname{SINR}_{s}>2^{r}-1\right\} \mathrm{d} r,
\end{aligned}
$$

which is equal to $(1-\epsilon) \lambda_{s} \mathcal{T}\left(y,(1-\epsilon) \lambda_{s}\right)$. Here, we solve the following optimization problem:

$$
G^{*}=\max _{\lambda_{s} \in \Lambda_{s}^{*}}(1-\epsilon) \lambda_{s} \mathcal{T}\left(y,(1-\epsilon) \lambda_{s}\right) .
$$

For the case that $\Lambda_{s}^{*}=[0, \infty)$, authors in [9] and [12] have derived the optimal solution. In this paper, we only study the spatial throughput of the SS through the simulations. Only different aspects of the above optimization versus the others formulated in the cases of power control mechanism and cooperative spectrum sensing are highlighted.

Let $\Lambda_{s}^{* P}, \Lambda_{s}^{* C}$, and $\Lambda_{s}^{* D}$ indicate the set of allowable secondary transmitter density with power control mechanism, centralized cooperative spectrum sensing, and decentralized cooperative spectrum sensing, respectively. For these cases, we have

$$
\begin{array}{r}
G^{* P}=\max _{\lambda_{s} \in \Lambda_{s}^{* P}}(1-\epsilon) \lambda_{s} e^{-\mu \frac{T}{S_{s}} y^{\alpha}} \mathcal{T}\left(y,(1-\epsilon) \lambda_{s} e^{-\mu \frac{T}{S_{s}} y^{\alpha}}\right), \\
G^{* D}=\max _{\lambda_{s} \in \Lambda_{s}^{* D}}\left(1-\epsilon^{D}\right) \lambda_{s} \mathcal{T}\left(y,\left(1-\epsilon^{D}\right) \lambda_{s}\right), \\
G^{* C}=\max _{\lambda_{s} \in \Lambda_{s}^{* C}}\left(1-\epsilon^{C}\right) \lambda_{s} \mathcal{T}\left(y,\left(1-\epsilon^{C}\right) \lambda_{s}\right),
\end{array}
$$

where $\epsilon^{C}$ and $\epsilon^{D}$ are, respectively, false alarm probabilities in centralized and decentralized spectrum sensing. Note that based on the support intervals $\Lambda_{s}^{* P}, \Lambda_{s}^{*}, \Lambda_{s}^{* C}$, and $\Lambda_{s}^{* D}$ and the amounts of $1-\epsilon,(1-\epsilon) \lambda_{s} e^{-\mu_{S_{s}}^{T} \alpha^{\alpha}}, 1-\epsilon^{C}$, and $1-\epsilon^{D}$, one of the above optimization problems may outperform the others.

Direct observation results in $\Lambda_{s}^{*} \subseteq \Lambda_{s}^{* P} \subseteq \Lambda_{s}^{* D} \subseteq \Lambda_{s}^{* C}$. In fact, from Proposition 5, we know that in AND-rule centralized spectrum, sensing $P_{\text {out }}^{p}=0$ for all secondary transmitter density $\lambda_{s}$, thus $\Lambda_{s}^{* C}=[0, \infty)$. Moreover, result of Proposition 6 indicates that for small and large $\lambda_{s}, P_{o u t}^{p}$ is sufficiently small. For example, it may form like $\Lambda_{s}^{* D}=$ $[0, a) \cup(b, \infty]$ for spatial density $b \geq a$. For large enough outage constraint $\xi$, there holds $\Lambda_{s}^{* D}=[0, \infty)$. However, for small enough outage constraint $\xi$, we have $\Lambda_{s}^{* D}=[0$, $\left.\lambda_{s}^{*}\right) \cup(b, \infty]$. Thus, it is seen that $\Lambda_{s}^{*} \subseteq \Lambda_{s}^{* D} \subseteq \Lambda_{s}^{* C}$. Note that PS outage probability with power control mechanism is larger than that of decentralized cooperative spectrum sensing for moderate and large $\lambda_{s}$ (see Fig. 12). So, we can conclude that $\Lambda_{s}^{*} \subseteq \Lambda_{s}^{* P} \subseteq \Lambda_{s}^{* D}$.

On the other hand, in the case of centralized cooperative spectrum sensing, miss detection probability is zero; thus, surfing the typical ROC, we have $\epsilon^{C}=1$. Notice that in centralized AND rule with $n$ collaborative secondary users,
TABLE 2

Simulation Parameters

\begin{tabular}{l|c}
\hline Parameter & Value \\
\hline Propagation loss exponent & 4 \\
Cell radius & $5000 \mathrm{~m}$ \\
$R$ & $20 \mathrm{~m}$ \\
$\mu$ & $8 \mathrm{~dB}$ \\
$\kappa$ & 1 \\
Background noise power $\left(N_{0} B\right)$ & $10^{-8} \mathrm{Watt}$ \\
$S_{s}$ & $0.1 \mathrm{Watt}$ \\
$S_{p}$ & $1 \mathrm{Watt}$ \\
$\mathrm{y}$ & $2 \mathrm{~m}$ \\
$\gamma_{t h}$ & $10 \mathrm{~dB}$ \\
\hline
\end{tabular}

$\epsilon^{C}=1-(1-\epsilon)^{n}$ approaches zero if $n \rightarrow \infty$, thus $G^{* C}=0$. In fact, with high probability, we can often find secondary transmitters that wrongly consider an idle spectrum as busy. This indicates that although centralized AND rule successfully vanishes any potential threats to primary receiver, its throughput performance is zero.

For the case of decentralized cooperative spectrum sensing and for given $\lambda_{s} \in \Lambda_{s}^{* D}$ following the same lines of argument as in Proposition 6, we suggest that $\epsilon^{D}=$ $1-(1-\epsilon) e^{-\epsilon \lambda_{s} \pi R_{c}^{2}}$. For $R_{c} \rightarrow \infty, \epsilon^{D}$ approaches 1 ; thus, $G^{* D} \rightarrow 0$.

The truncated power control (19) utilizes the randomness of the channel fading to transmit on good fading realizations. This results in reducing the PS outage probability. Thus, intuitively, there holds $\Lambda_{s}^{*} \subseteq \Lambda_{s}^{* P}$. Moreover, it is easy to see that $(1-\epsilon) \lambda_{s} e^{-\mu \frac{T}{S_{s}} y^{\alpha}} \leq(1-\epsilon) \lambda_{s}$. As a result, power control results in reducing the effective aggregated interference at each secondary receiver imposed by transmitting of the other secondary transmitters. Note that for each secondary link, the corresponding rate will be increased due to improving the attended received signal as well as decreasing the effect of aggregated interference. Consequently, we anticipate that power control can dramatically enhance the mean spatial of secondary throughput.

\section{Simulation Studies}

In this section, simulation results are compared to the obtained analytical derivations in this paper. In our simulations, we adopt the Monte Carlo method with sufficient number of iterations. The main simulation parameters are presented in Table 2 . The primary receiver is fixed and located at the origin. The number of the secondary users in the secondary network is the output of a Poisson distribution with mean value $\lambda_{s}$. Secondary users are uniformly distributed on a plane. To highlight the difference between the mathematical derivations and simulation results, the background noise power is considered higher than usual.

\subsection{PS Outage Probability}

Fig. 7 presents the PS outage probability versus the SS transmitters density, $\lambda_{s}$. Here, we assume that all the SS transmitters have the same ROC curve, we further fix $\delta=0.05$. In case of no SS transmitter, Fig. 7 indicates that the outage probability of the PS remains constant by increasing $\lambda_{s}$. As it is expected, in the presence of a SS network, it is also seen that by increasing $\lambda_{s}$, the outage probability of the PS is also increased. Indeed, by increasing 


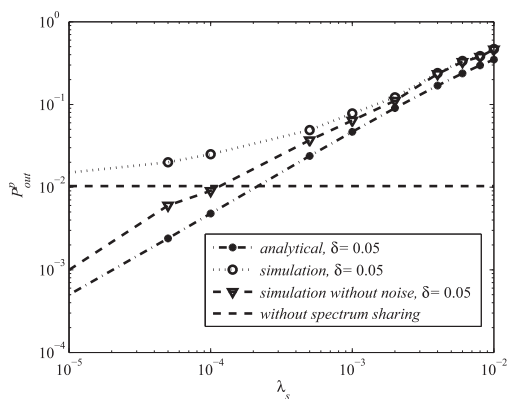

Fig. 7. The PS outage probability versus the density of the SS transmitters, $\lambda_{s}$, for $\delta=0.05, N_{0} B=0,10^{-8}$.

$\lambda_{s}$, the number of the SS transmitters with miss detection per unit area is increased.

In Fig. 7, we also compare analytical and simulation results. As it is seen, by considering the background noise power, there is a gap between the simulations and analytical results. This gap is mostly eliminated when $\lambda_{s}$ is increased. This is mainly due to considering a higher than usual background noise in the simulation. Note that in the derivations, the background noise was ignored. The gap is decreased since for higher $\lambda_{s}$, the share of the background noise in the total interference is decreased.

For the case where there is no spectrum sharing, it is seen that the analytical result is below the actual $P_{o u t}^{p}$. This is mainly due to ignoring the background noise. Therefore, this figure suggests that ignoring the background noise is acceptable only when the amount of $\lambda_{s}$ is sufficiently high. It must be noted that here, a disk with radius 5,000 meter is considered; however, the analytical derivations are obtained for $\mathbb{R}^{2}$, i.e., infinite radius. This is the reason that there is some differences between the analytical and simulation results for higher values of $\lambda_{s}$. Simulations results without background noise are also presented in Fig. 7 , in this case the analytical results follow the simulations closely.

\subsection{Path-Loss Singularity Phenomena}

As it was also mentioned in Section 2, the considered distance-dependent path-loss model gets infinite values by approaching the distance among some SS transmitters and the PS receiver to zero which is known as the path-loss singularity phenomena. Here, we show that this phenomena has only negligible impact on the analytical and simulation results presented in this paper. To do this, as [37] and [11], let us consider three different distance-dependent path-loss models: $l(x, y)=\|x-y\|^{-\alpha}, l(x, y)=(1+\|x-y\|)^{-\alpha}$, and $l(x, y)=(\max \{1,\|x-y\|\})^{-\alpha}$; we refer to these models as systems I, II, and III, respectively.

In Fig. 8, the PS outage probability versus the SS transmitter density is plotted for three different path-loss exponent. Note that in this illustration, the effect of ambient thermal noise is not considered. Therefore, the obtained results are not in contrast to the results presented in Fig. 6. As Fig. 8 also indicates, these three systems achieve almost the same PS outage probability as it was stated in Section $2 .{ }^{6}$

6. The results of this simulation are discussed in [16] as follows: For a transmitter receiver that are located very close to each other, the channel model in system I results in a very small SINR. However, the receiver would be very likely be in the outage even if the singularity was removed.

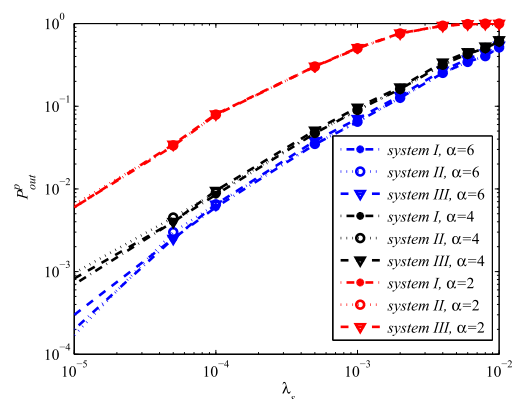

Fig. 8. The PS outage probability versus the density of the SS transmitters for three different path-loss models ignoring ambient noise effect.

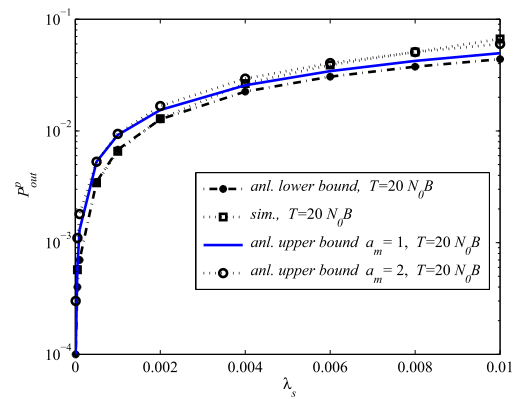

Fig. 9. The PS outage probability versus the density of the SS transmitters for systems with power control mechanism ignoring ambient noise effect.

\subsection{Impact of Power Control and Cooperative Sensing}

Fig. 9 demonstrates the PS outage probability in the case that the SS transmitters use the truncated channel inversion power control with $T=20 N_{0} B$. In this simulation, we plot the obtained approximation of the PS outage probability, $P_{\text {out }}^{p}\left(a_{m}(4)\right)$, for $a_{m}(4)=1,2$. As it is observed in this case, the simulated result and $P_{o u t}^{p}(1)$ are very close; therefore, $P_{\text {out }}^{p}(1)$ gives a tight approximation of the PS outage probability. Here, it is seen that the presented lower bound, $P_{\text {out }}^{l, p}$ and $P_{\text {out }}^{p}(2)$ are also tight enough to approximate the PS outage probability. As a result, the presented approach to approximate the PS outage probability studied in Section 4 is also applicable to the systems with power control.

Here, we verify the presented results for the cooperative spectrum sensing in Section 6. Fig. 10 presents the PS outage probability versus the SS transmitter density for the system including centralized and decentralized cooperative spectrum sensing. First, we consider the centralized case. Proposition 6 anticipates the density of the SS transmitter with miss detection experience approaches to zero in this case. Therefore, the PS outage probability actually reduces to the case that the aggregate interference at the PS receiver is zero. The simulation results also confirm our analytical results, although there is a gap between the analytical and simulation results that decreases to zero by increasing $\lambda_{s}$. For the case of the decentralized cooperative spectrum sensing, Fig. 10 also indicates that the analytical and simulation results follow almost the same pattern.

The impact of the cooperative spectrum sensing consisting the $M$-out-of- $N$ voting rule at the fusion center is illustrated in Fig. 11. 


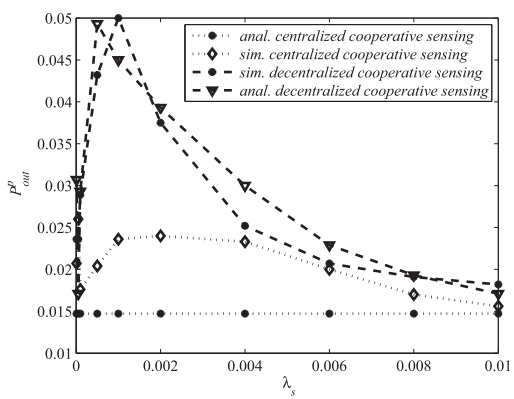

Fig. 10. The PS outage probability versus the density of the SS transmitters for systems with centralized and decentralized cooperative spectrum sensing.

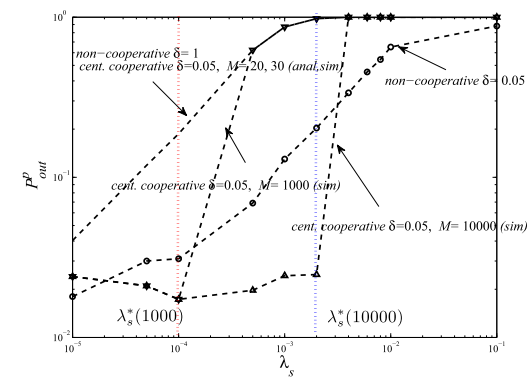

Fig. 11. The PS outage probability versus the density of the SS

In this figure, we present the PS outage probability versus the SS transmitter density considering different values of $M$. As it is observed, for the small values of $M$, $M \ll 1,000$, the $M$-out-of- $N$ voting rule puts the secondary system with miss detection probability $\delta<1$ at the same place as $\delta=1$. Using this rule, the data fusion identifies the spectrum as idle in cases where $M$ separate indicators are standing for the idle spectrum. Here, in the outage point of view, the secondary network acts similar to the case if all secondary users experience miss detection.

For large enough values of $M$, however, i.e., $M \geq 1,000$, Fig. 11 suggests a phase transition in the behavior of the PS outage probability. There is a critical $\lambda_{s}^{*}(M)$ where for $\lambda_{s} \leq$ $\lambda_{s}^{*}(M)$ the actual density of secondary transmitters with miss detection experience, $\lambda$ tends to zero. For the case that $\lambda_{s}>\lambda_{s}^{*}(M)$, the effectiveness of the spectrum sensing is completely vanishes, i.e., the density of the SS transmitters with miss detection experience approaches $\lambda_{s}$.

Fig. 12 shows the PS outage probability versus the SS transmitter density. As it is seen, by utilizing power control strategy, in this case the truncated channel inversion with $T=20 N_{0} B$, the PS outage probability is significantly decreased where the gap is increased by increasing $\lambda_{s}$. In this figure, we also plot the PS outage probability when the centralized cooperative spectrum sensing is adopted in the SS. As we expect in this case, the PS outage probability is reduced to the case that there is no spectrum sharing. For the decentralized cooperative spectrum sensing, two different behaviors are observed. For small enough $\lambda_{s}$, the outage probability follows the same pattern when cooperative sensing is not adopted. This is due the fact that there is not enough SS transmitters to establish an effective distributed cooperative sensing. However, for large enough $\lambda_{s}$, it is seen that the outage probability acts similar to the

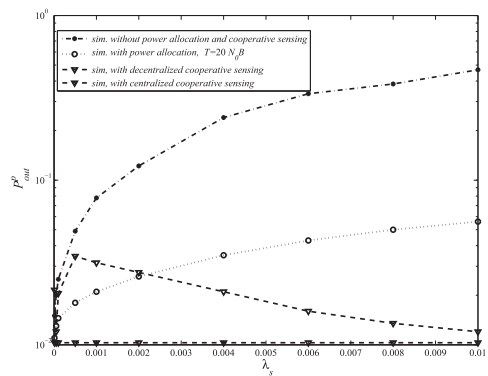

Fig. 12. The PS outage probability versus the density of the SS transmitters for systems with/without power control and centralized and decentralized cooperative spectrum sensing.

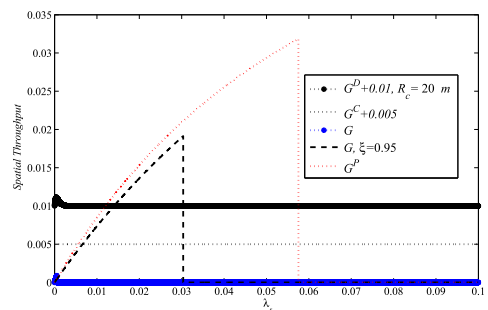

Fig. 13. The SS spatial throughput versus the density of the SS transmitters for system with/without power control and centralized and decentralized spectrum sensing ignoring ambient noise effect.

centralized cooperative spectrum sensing. Therefore, centralized and decentralized cooperative spectrum sensing achieve almost the same performance where the SS transmitters density is large enough.

\subsection{SS Performance}

In this section, we study the spatial throughput of the SS. As it was also mentioned in Section 7 in our analysis, we only focus on the mean spatial throughput obtained in correctly sensed spectrum holes.

Fig. 13 shows the impact of the density of the SS transmitters on the spatial throughput. Here, we set $\xi=0.05$. Note that for more clarity, we depict $G^{* C}+0.05$ and $G^{*} D+0.01$. As it can be seen for $\xi=0.05$, we have $G^{*}=0, G^{* C}=0$, and $G^{* D}=0$ almost for all vales of spatial density $\lambda_{s}$. We also depict $G^{*}$ for $\xi=0.95$.

In curve $G^{* P}$ by increasing $\lambda_{s}$, at first, spatial throughput is also increased. However, in this case, we have a sharp drop at about 0.06 which corresponds to the primary outage constraint $\xi=0.05$. Here, we have $\Lambda^{* P} \approx[0,0.06]$. Note that as it can be obviously seen for $\xi=0.05, \Lambda_{s}^{*}=\emptyset$ which indicates that SS without power control performs weakly. Even for the case of $\xi=0.95$, spatial throughput of the SS cannot reach the performance with power control.

For centralized and decentralized spectrum sensing, the false alarm probabilities are zero; thus, no mean spatial throughput is obtained. For better illustration, we also depict the case of $G^{*}$ and $G^{* D}$ in Fig. 14. As it can be seen for small values of $\lambda_{s}$, the decentralized cooperative spectrum sensing outperforms $G^{*}$. This is mainly due to the fact that the primary outage probability adopting cooperation is smaller than the latter.

Fig. 15 demonstrates the impact of $\xi$ on the mean spatial throughput of the SS. As it can be seen, the larger $\xi$ is, the larger $G^{*}$ will be. This is mainly because of expanding the 


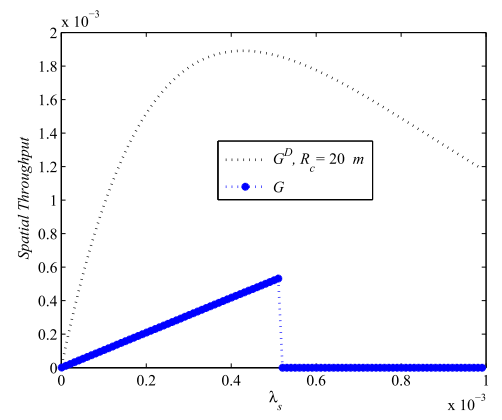

Fig. 14. The SS spatial throughput versus the density of the SS transmitters for system with/without decentralized spectrum sensing ignoring ambient noise effect.

interval $\Lambda^{*}$. Conducting power control mechanism, we find out that this event happens for small value of $\xi$; thus, for outage constraints larger than $0.1, G^{* P}$ stays almost constant. Indeed, for $\xi>0.05$ interval $\Lambda^{* P}$ always contains value of $\lambda^{*}$ that maximizes (30). This happens very soon in the case of decentralized cooperative spectrum sensing. Indeed, $G^{* D}$ so soon reaches its maximum; however, the amount of its maximum is too low compared to $G^{* P}$ due to small false alarm probability. For all $\xi$, we obviously have $G^{* C}=0$. For $G^{*}$ increasing $\xi$ increases its amount. This is due to expanding the support interval $\Lambda_{s}^{*}$.

\section{Conclusions}

In this paper, we utilized stochastic geometry to analyze the PS outage performance for spectrum sharing in Rayleigh fading environment. Using this approach, the impacts of the SS parameters and wireless environment on the PS outage probability were analyzed. We further obtained a closed form for the PS outage probability. The maximum SS transmitter node density for a given outage probability constraint of the PS was then calculated. The obtained maximum density of the SS transmitters extends our vision on the essential SS parameters that adjusting them enables us to guarantee the performance of the PS.

Moreover, we extended our analysis to the case that the secondary network is facilitated with a power control strategy and showed that even a simple power control mechanism, for example, truncated channel inversion significantly decreases the PS outage probability. Cases with centralized and decentralized AND-rule cooperative spectrum sensing were also considered, and their corresponding PS outage probabilities were also analyzed. It was shown that in the centralized cooperative spectrum sensing, the SS transmitters density with miss detection approached to zero. However, in the decentralized cooperative spectrum sensing by increasing the SS transmitters density, the negative impact of the SS on the PS is decreased. Simulations results confirmed our analytical derivations.

We also studied issues about the SS mean spatial throughput. We investigated the impact of the secondary transmitter density and the PS outage constraint of the spatial throughput on the SS. Moreover, truncated power control mechanism was able to enhance the SS spatial throughput.

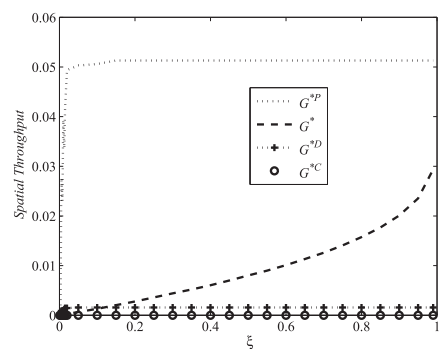

Fig. 15. SS spatial throughput versus the PS outage constraint for system with/without power control and centralized and decentralized spectrum sensing ignoring ambient noise.

\section{ACKNOWLEDGMENTS}

Part of this paper was presented at the IEEE ICC 2010, May 2010. This work was partly supported by the UK Engineering and Physical Sciences Research Council (EPSRC) under grant EP/K/022725/1 and European Research Council CIG grant 304084 S3isE.

\section{REFERENCES}

[1] "Spectrum Policy Taskforce Report," technical report, Fed. Comm. Commission, Nov. 2002.

[2] J.M. Peha, "Approaches to Spectrum Sharing," IEEE Comm. Magazine, vol. 43, no. 2, pp. 10-12, Feb. 2005.

[3] S. Haykin, "Cognitive Radio: Brain-Empowered Wireless Communications," IEEE J. Selected Areas in Comm., vol. 23, no. 2, pp. 201-220, Feb. 2005.

[4] Q. Zhao and B. Sadler, "A Survey of Dynamic Spectrum Access: Signal Processing, Networking, and Regulatory Policy," IEEE Signal Processing Magazine, vol. 24, no. 3, pp. 79-89, May 2007.

[5] M.G. Khoshkholgh, K. Navaie, and H. Yanikomeroglu, "Access Strategies for Spectrum Sharing in Fading Environment: Overlay, Underlay and Mixed," IEEE Trans. Mobile Computing, vol. 9, no. 12, pp. 1780-1793, Dec. 2010.

[6] X. Liu and S.N. Shankar, "Sensing-Based Opportunistic Channel Access," ACM J. Mobile Networks and Applications, vol. 11, no. 4, pp. 577-591, Aug. 2006.

[7] J.F.C. Kingman, Poisson Processes. Oxford Univ., 1993.

[8] E.S. Sousa and J.A. Silvester, “Optimum Transmission Ranges in a Direct-Sequence Spread-Spectrum Multihop Packet Radio Network," IEEE J. Selected Areas in Comm., vol. 8, no. 5, pp. 762-771, June 1990.

[9] F. Baccelli et al., "An ALOHA Protocol for Multihop Mobile Wireless Networks," IEEE Trans. Information Theory, vol. 52, no. 2, pp. 421-436, Feb. 2006.

[10] C.C. Chan and S.V. Hanly, "Calculating the Outage Probability in a CDMA Network with Spatial Poisson Traffic," IEEE Trans. Vehicular Technology, vol. 50, no. 1, pp. 183-204, Jan. 2001.

[11] R.K. Ganti and M. Haenggi, "Interference and Outage in Clustered Wireless Ad Hoc Networks," IEEE Trans. Information Theory, vol. 55, no. 9, pp. 4067-4086, Sept. 2009.

[12] F. Baccelli et al., "Stochastic Analysis of Spatial and Opportunistic ALOHA," IEEE J. Selected Areas in Comm., vol. 27, no. 7, pp. 11051119, Sept. 2009

[13] M. Haenggi et al., "Stochastic Geometry and Random Graphs for the Analysis and Design of Wireless Networks," IEEE J. Selected Areas in Comm., vol. 27, no. 7, pp. 1029-1046, Sept. 2009.

[14] M. Haenggi and R.K. Ganti, "Interference in Large Wireless Networks," Foundations and Trends in Networking, vol. 3, no. 2, pp. 127-248, 2008.

[15] S.P. Weber et al., "Transmission Capacity of Wireless Ad Hoc Networks with Outage Constraints," IEEE Trans. Information Theory, vol. 51, no. 12, pp. 4091-4102, Dec. 2005.

[16] S.P. Weber et al., "The Effect of Fading, Channel Inversion, and Threshold Scheduling on Ad Hoc Networks," IEEE Trans. Information Theory, vol. 53, no. 11, pp. 4127-4149, Nov. 2007.

[17] S.P. Weber et al., "Transmission Capacity of Wireless Ad Hoc Networks with Successive Interference Cancellation," IEEE Trans. Information Theory, vol. 53, no. 8, pp. 2799-2814, Aug. 2007. 
[18] O. Dousse et al., "Impact of Interferences on Connectivity in Ad Hoc Networks," IEEE/ACM Trans. Networking, vol. 13, no. 2, pp. 425-436, Apr. 2005.

[19] S.J. Baek et al., "Minimizing Energy Consumption in Large-Scale Sensor Networks through Distributed Data Compression and Hierarchical Aggregation," IEEE J. Selected Areas in Comm., vol. 22, no. 6, pp. 1130-1140, Aug. 2004.

[20] S.J. Baek and G. de Veciana, "Spatial Model for Energy Burden Balancing and Data Fusion in Sensor Networks Detecting Bursty Events," IEEE Trans. Information Theory, vol. 53, no. 10, pp. 36153628, Oct. 2007.

[21] D. Zhang et al., "Spatial Capacity of Narrowband vs. UltraWideband Cognitive Radio Systems," IEEE Trans. Wireless Comm., vol. 7, no. 11, pp. 4670-4680, Nov. 2008.

[22] R. Menon et al., "On the Impact of Dynamic Spectrum Sharing Techniques on Legacy Radio Systems," IEEE Trans. Wireless Comm., vol. 7, no. 11, pp. 4198-4207, Nov. 2008.

[23] A. Ghasemi and E.S. Sousa, "Interference Aggregation in Spectrum-Sensing Cognitive Wireless Networks," IEEE J. Selected Topics in Signal Processing, vol. 2, no. 1, pp. 41-56, Feb. 2008.

[24] Y. Wen et al., "The Impact of Fading on the Outage Probability in Cognitive Radio Networks," Proc. IEEE Vehicular Technology Conf., pp. 1-5, Sept. 2010.

[25] Y. Wen et al., "On Distribution of Aggregate Interference in Cognitive Radio Networks," Proc. 25th Biennial Symp. Comm. (QBSC), pp. 265-268, May 2010.

[26] V. Mordachev and S. Loyak, "On Node Density-Outage Probability Tradeoff in Wireless Networks," IEEE J. Selected Areas in Comm., vol. 27, no. 7, pp. 1120-1131, Sept. 2009.

[27] C.H. Lee and M. Haenggi, "Interference and Outage in Doubly Poisson Cognitive Networks," Proc. Int'l Conf. Computer Comm. and Networks (ICCCN '10), pp. 1-6, Aug. 2010.

[28] K. Huang et al., "Spectrum Sharing between Cellular and Mobile Ad Hoc Networks: Transmission-Capacity Trade-Off," IEEE J. Selected Areas in Comm., vol. 27, no. 7, pp. 1256-1267, Sept. 2009.

[29] W. Ren et al., "Power Control in Cognitive Radio Networks: How to Cross a Multi-Lane Highway," IEEE J. Selected Areas in Comm., vol. 27, no. 7, pp. 1283-1296, Sept. 2009.

[30] A.J. Goldsmith, Wireless Communications. Cambridge Univ., 2005.

[31] M.G. Khoshkholgh, K. Navaie, and H. Yanikomeroglu, "On the Impact of the Primary Network Activity on the Achievable Capacity of Spectrum Sharing over Fading Channels," IEEE Trans. Wireless Comm., vol. 8, no. 4, pp. 2100-2111, Apr. 2009.

[32] R.H. Etkin, "Spectrum Sharing: Fundamental Limits, Scaling Laws, and Self-Enforcing Protocols," PhD dissertation, Electrical Eng. and Computer Sciences, Univ. of California at Berkeley, Dec. 2006.

[33] A.M. Hunter et al., "Transmission Capacity of Ad Hoc Networks with Spatial Diversity," IEEE Trans. Wireless Comm., vol. 7, no. 12, pp. 5058-5071, Dec. 2008.

[34] Q. Chen et al., "Cooperative Spectrum Sensing Strategies for Cognitive Radio Mesh Networks," IEEE J. Selected Topics in Signal Processing, vol. 5, no. 1, pp. 56-67, Feb. 2011.

[35] T. Yucek and H. Arslan, "A Survey of Spectrum Sensing Algorithms for Cognitive Radio Applications," IEEE Comm. Surveys and Tutorials, vol. 11, no. 1, pp. 116-130, First Quarter 2009.

[36] J.G. Andrews et al., "A Tractable Approach to Coverage and Rate in Cellular Networks," IEEE Trans. Comm., vol. 59, no. 11, pp. 3122-3134, Nov. 2011.

[37] H. Inaltekin et al., "On Unbounded Path-Loss Models: Effects of Singularity on Wireless Network Performance," IEEE J. Selected Areas in Comm., vol. 27, no. 7, pp. 1078-1092, Sept. 2009.

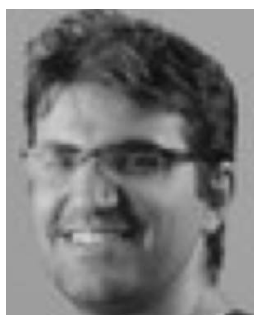

Mohammad G. Khoshkholgh received the BSc degree in electrical engineering from Isfahan University, Iran, in 2006 and the MSc degree in electrical engineering from Tarbiat Modares University, Tehran, Iran, in 2008. He is currently working toward the $\mathrm{PhD}$ degree at the Simula School of Research and Innovation, Oslo, Norway. His research interests are mainly in wireless communications, radio resource allocations, and spectrum sharing.

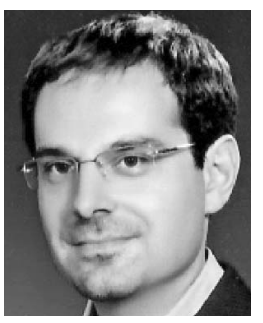

Keivan Navaie received the BSc degree from the Sharif University of Technology, Tehran, Iran, the MSc degree from the University of Tehran, Iran, and the PhD degree from Tarbiat Modares University, Tehran, Iran, all in electrical engineering, in 1995, 1997, and 2004, respectively. From March to November 2004, he was with the School of Mathematics and Statistics, Carleton University, Ottawa, Canada, as a postdoctoral research fellow. From December 2004 to September 2006, he was with the Broadband Communication and Wireless System (BCWS) Centre, Carleton University, Ottawa, Canada, where he was the project manager of BCWS participation in European Union 6th Framework integrated project, the Wireless World Initiative New Radio (WINNER) on beyond $3 \mathrm{G}$ wireless systems. From September 2006 to July 2011, he was with the Department of Electrical and Computer Engineering, Tarbiat Modares University, Tehran, Iran. Since July 2011, he has been at the School of Electrical and Computer Engineering, University of Leeds, United Kingdom. His research interests lie in the field of radio resource allocation for wireless communication systems, dynamic spectrum allocation, cognitive radio networks, and cooperative communications. He is on the editorial board of the European Transactions on Telecommunications. He has been on the technical program committee of different IEEE conferences, including GlobeCom, ICC, VTC, and WCNC, and chaired some of their symposia. He has also served as cochair of Wireless Network Track, IEEE VTC 2012, Yokohama, Japan, and the IEEE Eighth International Workshop on Wireless Network Measurements WiNMee 2012, Paderborn, Germany. He received the 2011 IEEE Iran Section Young Investigator Award. $\mathrm{He}$ is a senior member of the IEEE.

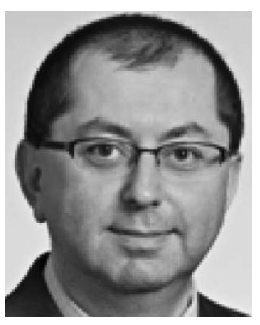

Halim Yanikomeroglu received the BSc degree in electrical and electronics engineering from the Middle East Technical University, Ankara, Turkey, in 1990, the MASc degree in electrical engineering (now ECE), and the PhD degree in electrical and computer engineering from the University of Toronto, Canada, in 1992 and 1998, respectively. He was with the R\&D Group of Marconi Kominikasyon A.S., Ankara, Turkey, from 1993-1994. Since 1998, he has been with the Department of Systems and Computer Engineering at Carleton University, Ottawa, where he is currently a full professor. His research interests cover many aspects of the physical, medium access, and networking layers of wireless communications with a special emphasis on the next-generation cellular system. In recent years, his research has been funded by Huawei (Canada and China), Research In Motion (Canada), Samsung Advanced Institute of Technology (Korea), Communications Research Centre of Canada, Nortel, Natural Sciences and Engineering Research Council of Canada, and Carleton University. He received the Carleton University Faculty Graduate Mentoring Award in 2010, the Carleton University Graduate Students Association Excellence Award in Graduate Teaching in 2010, and the Carleton University Research Achievement Award in 2009. He is a distinguished lecturer for the IEEE Vehicular Technology Society. He has been involved in the steering committees and technical program committees of numerous international conferences in communications; he has also given about 20 tutorials in such conferences. He is a member of the steering committee of the IEEE Wireless Communications and Networking Conference (WCNC) and has been involved in the organization of this conference over the years, including serving as the technical program cochair of WCNC 2004 and the technical program chair of WCNC 2008; he will also serve as the technical program cochair of WCNC 2014 to be held in Istanbul. He was the general cochair of the IEEE Vehicular Technology Conference Fall 2010 held in Ottawa. $\mathrm{He}$ is an editor for IEEE Transactions on Communications. He was an editor for the IEEE Transactions on Wireless Communications and IEEE Communications Surveys and Tutorials. He was the chair of the IEEE's Technical Committee on Personal Communications (now called, Wireless Technical Committee). During the 2011-2012 academic year, he was at the TOBB University of Economics and Technology, Ankara, Turkey, as a visiting professor. He is also an adjunct professor at King Saud University's Advanced Technology Research Institute, Riyadh, Saudi Arabia. He is a registered professional engineer in the province of Ontario, Canada. $\mathrm{He}$ is a senior member of the IEEE. 